Research Article

\title{
Biocompatibility and Angiogenic Effect of Chitosan/Graphene Oxide Hydrogel Scaffolds on EPCs
}

\author{
Lifang Zhang $\mathbb{D}^{\mathbb{1}},{ }^{1}$ Xinping Li, ${ }^{1}$ Congying Shi $\mathbb{D}^{\mathrm{D}},{ }^{2}$ Gaoying Ran, ${ }^{1}$ Yuting Peng, \\ Shuguang Zeng $\mathbb{D}^{1}{ }^{1}$ and Yan $\mathrm{He}^{3}$ \\ ${ }^{1}$ Department of Oral and Maxillofacial Surgery, Stomatological Hospital, Southern Medical University, 510000 Guangzhou, \\ Guangdong, China \\ ${ }^{2}$ Clinical Application Center, Guangdong Cord Bank, 510000 Guangzhou, Guangdong, China \\ ${ }^{3}$ Skeletal Biology Research Center, Department of Oral Maxillofacial Surgery, Harvard School of Dental Medicine, Boston, \\ 02114 MA, USA
}

Correspondence should be addressed to Shuguang Zeng; sunrisezdoctor@smu.edu.cn

Received 28 February 2021; Revised 29 March 2021; Accepted 22 April 2021; Published 19 May 2021

Academic Editor: Jing Yan

Copyright ( 92021 Lifang Zhang et al. This is an open access article distributed under the Creative Commons Attribution License, which permits unrestricted use, distribution, and reproduction in any medium, provided the original work is properly cited.

\begin{abstract}
Angiogenesis in the field of tissue engineering has attracted significant attention. Graphene oxide has become a promising nanomaterial in tissue engineering for its unique biochemical properties. Therefore, herein, a series of chitosan (CS)/graphene oxide (GO) hydrogel scaffolds were synthesized by crosslinking CS and GO at different concentrations (0.1, 0.5, and 1.0 wt.\%) using genipin. Compared with the CS hydrogel scaffolds, the CS/GO hydrogel scaffolds have a better network structure and mechanical strength. Then, we used endothelial progenitor cells (EPCs) extracted from human umbilical cord blood and cocultured these EPCs with the as-prepared scaffolds. The scaffolds with 0.1 and $0.5 \mathrm{wt} \% \mathrm{GO}$ showed no considerable cytotoxicity, could promote the proliferation of EPCs and tube formation, and upregulated the expressions of CD34, VEGF, MMP9, and SDF-1 in EPCs compared to the case of the scaffold with $1.0 \mathrm{wt} \% \mathrm{GO}$. This study shows that the addition of graphene oxide improves the structure of chitosan hydrogel and enhances the proliferation activity and angiogenic capacity of EPCs.
\end{abstract}

\section{Introduction}

Tissue defects caused by trauma, infection, and tumor resection have become a serious problem in healthcare worldwide [1]. Although surgery is the most valid method to treat these defects, it has certain limitations. In this regard, tissue engineering has attracted significant attention. However, as tissue regeneration relies on blood vessels for the transport of nutrients and metabolic wastes, engineered tissues cannot be widely used because they lack a vascular system [2]. Without perfusion from implanted microvasculature, the thickness of engineered tissue constructs in vitro is limited to approximately $200 \mu \mathrm{m}$, which is the oxygen diffusional limit [3]. Therefore, sufficient angiogenesis can promote the survival and repair of damaged tissues [4]. Although several studies have been reported on the osteogenesis of scaffold materials, studies on the angiogenesis of these materials are in their infancy at present $[5,6]$. Hence, researchers in the field of tissue engineering have started to focus on improving the angiogenesis of tissue-engineered structures $[7,8]$.

A three-dimensional porous structure is conducive to the growth of endothelial cells and the formation of vascular structures $[9,10]$. Hydrogels, as a three-dimensional porous structure, acquire good biocompatibility, degradability, and suitable mechanical properties, and it can be developed and improved to achieve more potential scaffold materials [11, 12]. Chitosan (CS) is a deacetylated product of chitin, which has the advantages of widespread availability and nontoxicity [13]. Hydrogels composed of CS have good biocompatibility and degradability and are widely used as scaffold materials for tissue engineering $[14,15]$. However, the weak mechanical strength and low bone conductivity of CS limit the 
application of these scaffold materials in tissue engineering [16]. To overcome these limitations, many researchers have developed CS composite materials $[17,18]$ by incorporating inorganic bioactive materials, such as $\beta$-tricalcium phosphate and hydroxyapatite (HA), using CS to promote bone regeneration $[19,20]$. However, the application of these materials is hindered by their insufficient osteoinduction and angiogenesis [21].

In addition, graphene derivatives have gradually become a research hotspot in the field of nanobiomedicine as they can provide a favorable physical and chemical environment for bone formation [22]. Graphene oxide (GO) has shown considerable potential for biomedical applications, including bone tissue engineering, due to its excellent physical and chemical properties and biological activity [23, 24]. GO at an appropriate concentration can induce angiogenesis without significant cytotoxicity $[25,26]$. Moreover, GO has abundant oxygen-containing groups, which can increase the deposition of HA [27]. Many researchers have introduced GO into tissue-engineered implants and found that GO is effective in promoting angiogenesis [27-29]. Thus, the addition of GO nanoparticles to the CS hydrogel can overcome the abovementioned limitations of this hydrogel.

In addition to having a suitable three-dimensional scaffold, the growth and proliferation of seed cells are crucial. Endothelial progenitor cells (EPCs), which are precursors of endothelial cells, can stimulate the growth of collateral vessels in ischemic tissues and thus promote angiogenesis [30]. EPCs can be noninvasively obtained from umbilical cord blood and adult peripheral blood, thereby eliminating immunogenicity [31]. Therefore, the angiogenic capacity of a tissueengineered implant can be enhanced by introducing EPCs into it.

Furthermore, genipin (GNP) is a nontoxic, easily degradable natural substance, which has been widely used for crosslinking biological materials [32]. It was predicted that CS can react with genipin by nucleophilic ring-opening reactions, which is more conducive to the formation of spatial network structures in studies $[33,34]$. Several studies have shown that the cytotoxicity of GNP is significantly lower than that of the classical crosslinking agent glutaraldehyde in vitro [35], and GNP has been continuously applied to manufacture biopolymer scaffolds in bone tissue engineering [36, 37].

Accordingly, in this study, a series of CS/GO hydrogel scaffolds were fabricated by crosslinking CS and GO at different concentrations using GNP. These hydrogel scaffolds exhibited significantly improved stability. Subsequently, we investigated the physical and chemical properties of these scaffolds. Moreover, the angiogenic potential of these scaffolds was evaluated by coculturing them with human EPCs, and the possible mechanisms have been discussed.

\section{Materials and Methods}

2.1. Hydrogel Production. Herein, $2.0 \mathrm{~g}$ CS (degree of deacetylation $\geq 95 \%$, Macklin, Shanghai, China) was dissolved in $100 \mathrm{~mL}$ aqueous hydrochloric acid $(0.1 \mathrm{~mol} / \mathrm{L}$, Sigma-Aldrich, St. Louis, State of Missouri, USA) under sterile conditions. The $\mathrm{pH}$ of the resulting solution was adjusted to approximately 7.25 by $\beta$-glycerophosphate sodium solution (70\% $w / v$, Solarbio, Beijing, China). Then, appropriate amounts of GNP solution ( $1 \% w / v$, Sigma-Aldrich) and GO aqueous solution $(3 \mathrm{mg} / \mathrm{mL}$, Macklin) were added to the abovementioned solution. By mechanical stirring and ultrasonic breaking, CS/GO mixed solutions with different GO concentrations were prepared. Thereafter, these mixed solutions were centrifuged at $4^{\circ} \mathrm{C}$ and $3000 \mathrm{r} / \mathrm{min}$ for $10 \mathrm{~min}$ and then placed in a biochemical incubator at $37^{\circ} \mathrm{C}$ to form the hydrogels. Finally, these hydrogels were frozen at $-80^{\circ} \mathrm{C}$ for $4 \mathrm{~h}$ and then placed in a vacuum freeze dryer (Biobase, Jinan, Shandong province, China) to construct hydrogel scaffolds.

2.2. Scanning Electron Microscopy (SEM). All lyophilized hydrogel scaffolds were cut into cubes with a side length of $0.5 \mathrm{~cm}$ followed by sputter coating with gold in liquid nitrogen. The internal structure and morphology of the scaffold sections were examined by SEM (Hitachi S-3700N, Chiyoda, Tokyo). Three samples of each group were chosen, and pictures were taken under an electron microscope. Three areas of each sample were selected randomly and measured by ImageJ. Typically, the average pore size was calculated.

2.3. Fourier Transform Infrared Spectroscopy (FTIR). Hydrogel scaffolds in appropriate amounts were ground and mixed with $\mathrm{KBr}$ (Macklin) particles in a 1:300 ratio. Then, they were scanned 64 times using a Fourier transform infrared spectrometer (TENSOR 27, Bruker, Karlsruhe, Baden-Wurttemberg, Germany) in the range of $4000-400 \mathrm{~cm}^{-1}$ at a resolution of $2 \mathrm{~cm}^{-1}$, and Fourier transform infrared spectra were recorded.

2.4. X-Ray Powder Diffraction (XRD). The abovementioned samples were analyzed using an X-ray diffractometer (D8X, Bruker), and the corresponding $\mathrm{XRD}$ patterns were acquired. The scanning area was in the $2 \theta$ range of $0-40^{\circ}$, and the scanning speed was $2^{\circ} / \mathrm{min}$.

2.5. Tensile Properties. Lyophilized scaffolds were cut into 2 $\mathrm{cm} \times 0.5 \mathrm{~cm}$ strips, and their tensile properties were measured using an electronic universal material testing machine (Qingji Instrument Technology Co., Ltd, Shanghai, China) and a $100 \mathrm{~N}$ load cell at a loading rate of $10 \mathrm{~mm} / \mathrm{min}$. The measurement was performed five times, and the average value was calculated.

2.6. Swelling Rate (SR). Suitable amounts of lyophilized scaffolds were weighed, immersed in a large amount of ultrapure water at $25^{\circ} \mathrm{C}$ for $24 \mathrm{~h}$, and then weighed again after removing the surface moisture:

$$
\mathrm{SR}=100 \% \times \frac{\mathrm{Ws}-\mathrm{Wd}}{\mathrm{Wd}}
$$

where Ws and Wd are the weights of the wet and dry hydrogel scaffolds, respectively.

2.7. Porosity (ع) Test. The specific method was as follows [38]: hydrogels (dry mass M0) were immersed in ethanol in a 
weighing bottle. The bottle was weighed before (Ma) and after $(\mathrm{Mb})$ removing the wet scaffold. Meanwhile, a $50 \mathrm{~mL}$ pycnometer filled with ethanol was weighed with the weight denoted as M1. The ethanol was poured out, and the wet scaffold previously soaked in ethanol was placed in the pycnometer, and ethanol was added until the pycnometer was filled to the same mark. The pycnometer was weighed again with weight denoted as M2. Porosity was calculated according to the following equations ( $\rho$ is the density of ethanol):

$$
\begin{aligned}
\mathrm{V} 1= & (\mathrm{Ma}-\mathrm{Mb}-\mathrm{M} 0) / \rho, \\
\mathrm{V} 2= & {[(\mathrm{Ma}-\mathrm{Mb})-(\mathrm{M} 2-\mathrm{M} 1)] / \rho, } \\
\mathcal{E}= & {[\mathrm{V} 1 / \mathrm{V} 2] \times 100 \%=(\mathrm{Ma}-\mathrm{Mb}-\mathrm{M} 0) /[(\mathrm{Ma}-\mathrm{Mb})} \\
& -(\mathrm{M} 2-\mathrm{M} 1)] \times 100 \%,
\end{aligned}
$$

where V1 is the volume of scaffold pores, V2 is the apparent volume of the scaffold, and $\varepsilon$ is the porosity of the scaffold. Measurements were repeated three times for the same sample, and the average value was obtained.

\subsection{Cell Subculture}

2.8.1. Subculture of EPCs. After the EPCs (purchased from the Guangdong Cord Blood Bank, Guangzhou, Guangdong province, China) adhere to the wall, the medium was changed every two days. The cells can be subcultured when they have grown to $80 \%$. After removing the original medium, rinse with PBS (Thermo Fisher Scientific, Waltham, Massachusetts, USA) twice and add $0.5 \mathrm{~mL} 0.25 \%$ trypsin (SigmaAldrich). When the cells are round, add medium to stop the digestion. After centrifugation at $800 \mathrm{rpm}$ for $3 \mathrm{~min}$, resuspension with Endothelial Cell Growth Medium (EGM) was used to inoculate in a culture flask and then cultured in a $37^{\circ} \mathrm{C}, 5 \% \mathrm{CO}_{2}$ incubator (Thermo Fisher Scientific).

2.9. Cell Identification. Herein, $1 \times 10^{6}$ EPCs were collected, and fluorescent dyes were used to conjugate cell surface markers: CD45/FITC, CD14/PE, CD34/PE, CD133/PE, CD31/FITC, CD105/FITC, and KDR-PE (Thermo Fisher Scientific). Cells were fixed with $4 \%$ paraformaldehyde (Beyotime Biotechnology). After being stained and washed with flow cytometry staining buffer (PBS $\left.+1 \% \mathrm{BSA}+0.1 \% \mathrm{NaN}_{3}\right)$, the cells were analyzed using a flow cytometer (BD Biosciences, Franklin Lakes, New Jersey, USA).

2.10. Tube Formation Experiment. Matrigel (Corning, New York, USA) was melted at $4^{\circ} \mathrm{C}$ and added to a 96-well plate precooled at $-20^{\circ} \mathrm{C}$ followed by the removal of air bubbles. Then, this plate was placed in a cell incubator at $37^{\circ} \mathrm{C}$ for $1 \mathrm{~h}$. The EPCs were treated with serum-free Endothelial Cell Growth Medium (EGM) for $1 \mathrm{~h}$ and then placed on a 96-well plate covered with Matrigel at $5 \times 10^{4}$ cells/well; thereafter, this plate was placed in a $37^{\circ} \mathrm{C}, 5 \% \mathrm{CO}_{2}$ humidified incubator. After $8 \mathrm{~h}$, tube formation was observed using a microscope (Olympus IX51, Japan), and images were acquired.
2.11. Cocultivation and Grouping of EPCs and Hydrogel Scaffolds. Hydrogel scaffolds were placed in a well plate, and then, an appropriate amount of EPCs was inoculated into these scaffolds according to the experimental requirements. Based on the concentration of GO in the scaffolds, the coculture system was divided into five groups: EPCs, CS; EPCs, CS/0.1 wt.\%GO; EPCs, CS/0.5 wt.\%GO; EPCs, $\mathrm{CS} / 1.0 \mathrm{wt} \% \mathrm{GO}$; and EPCs.

2.12. Cell Counting Kit-8 (CCK-8) Assay. Scaffolds in appropriate amounts were fabricated under aseptic conditions and spread on a 24 -well plate; then, $500 \mathrm{~mL}$ of a medium containing $5 \times 10^{4}$ EPCs was seeded in each well, and $50 \mu \mathrm{L}$ CCK-8 solution (Dojindo, Kumamoto, Japan) was added to each well every $36 \mathrm{~h}$. After incubation at $37^{\circ} \mathrm{C}$ for $2 \mathrm{~h}$, $100 \mu \mathrm{L}$ medium was withdrawn and added to a $96-$ well plate for absorbance measurement at $450 \mathrm{~nm}$ using a microplate reader (Bio-Rad, Hercules, California, USA). In each test, three duplicate wells were set for all specimens.

2.13. Lactate Dehydrogenase (LDH) Toxicity Assay. Scaffolds were synthesized under aseptic conditions and spread in 96well plates. The EPCs were digested and seeded at $1 \times 10^{4}$ cells/well. After 1, 3, and 5 days, three wells of cells for each group were centrifuged at $400 \mathrm{~g}$ for $5 \mathrm{~min}$, and the supernatants were obtained. LDH working solution (Beyotime Biotechnology) was prepared according to the instructions of the $\mathrm{LDH}$. The $\mathrm{LDH}$ working solution was completely mixed with the test solution. Approximately $120 \mu \mathrm{L}$ of supernatant was withdrawn from each well and put in a new 96-well plate. At room temperature, the 96-well plate wrapped with aluminum foil was placed on a horizontal shaker for slow shaking followed by incubation in the dark for $30 \mathrm{~min}$. Then, the absorbance was measured at $490 \mathrm{~nm}$ using the abovementioned microplate reader.

2.14. Live/Dead Staining. The EPCs were seeded on 12-well plates at $5 \times 10^{5}$ cells/well. After culturing in a cell incubator, the culture solution was withdrawn and gently soaked three times with PBS to ensure the removal of the active esterase present in the culture medium.

2.14.1. Configuration of the Staining Working Solution (Beyotime Biotechnology). Calcein and propidium iodide were equilibrated at room temperature for $30 \mathrm{~min}$, and the staining working solution was configured according to the instructions. The staining working solution $(200 \mu \mathrm{L})$ was added to each well to cover the bottom of the well plate, followed by incubation in the dark for $30 \mathrm{~min}$ and gentle washing three times with PBS for 5 min each time. The green and red fluorescence of the same region was examined using a fluorescence microscope (Olympus IX51, Japan).

2.14.2. Measurement of the Number of Dead and Live Cells. Trypsin without ethylenediaminetetraacetic acid was used to digest the previously seeded and soaked EPCs, followed by centrifugation and washing twice with PBS to resuspend the cells. Then, the cells of each group were stained by the abovementioned process followed by washing and 
TABLE 1: Sequences of primers for qRT-PCR.

\begin{tabular}{lcc}
\hline Gene symbol & Forward primer $\left(5^{\prime}-3^{\prime}\right)$ & Reverse primer $\left(5^{\prime}-3^{\prime}\right)$ \\
\hline CD 34 & AGACTGTGCAGTGATGTGGT & CCCTGGTACATTCGGGTCTG \\
$M M P 9$ & CCTGGGCAGATTCCAAACCT & GTACACGCGAGTGAAGGTGA \\
$V E G F$ & GGCAAAAACGAAAGCGCAAG & GAGGCTCCAGGGCATTAGAC \\
SDF- 1 & TGCCCTTCAGATTGTAGCCC & GCGTCTGACCCTCTCACATC \\
GAPDH & GGAGTCCACTGGCGTCTTCA & GTCATGAGTCCTTCCACGATACC \\
\hline
\end{tabular}

centrifugation with PBS. Finally, the measurement was conducted using the flow cytometer.

2.15. Tube Formation Assay. Cells were inoculated according to the instructions provided on the CCK-8 kit and continuously cultured for 7 days. The Matrigel basement membrane matrix (BD Biosciences, Franklin Lakes, New Jersey, USA) was thawed at $4^{\circ} \mathrm{C}$, mixed with the basal medium in equal proportion, pipetted into precooled 96-well plates, and incubated at $37^{\circ} \mathrm{C}$ for $30 \mathrm{~min}$. After Matrigel polymerization, EPCs were treated with serum-free EGM for $1 \mathrm{~h}$ and then digested and reseeded on Matrigel in 96-well plates at $1 \times$ $10^{4}$ cells/well. After being incubated at $37^{\circ} \mathrm{C}$ for $8 \mathrm{~h}$, the cells were imaged using a microscope (Leica, Wetzlar, HesseDarmstadt, Germany), and the images were analyzed and interpreted using ImageJ software.

2.16. Quantitative Reverse Transcription Polymerase Chain Reaction ( $q R T-P C R)$. The EPCs were seeded in 6-well plates at $1 \times 10^{6}$ cells/well and continuously cultured for 7 days. Total RNAs of the cells of each group were extracted with the TRIzol reagent (Sigma, Missouri, USA) and reverse transcribed into cDNA using an Evo M-MLV RT Premix kit (Accurate Biology, Changsha, Hunan, China). The SYBR ${ }^{\circledR}$ Green Premix Pro Taq HS qPCR Kit (Accurate Biology, Changsha, Hunan, China) was used for PCR amplification, and the total volume of the reaction system was $20 \mu \mathrm{L}$. PCR was performed employing the ABI PRISM ${ }^{\circledR} 7500$ sequence detection system. The reaction conditions were as follows: $95^{\circ} \mathrm{C}$ for $30 \mathrm{~s}$, followed by 40 cycles of $95^{\circ} \mathrm{C}$ for $5 \mathrm{~s}$ and $60^{\circ} \mathrm{C}$ for $30 \mathrm{~s}$. Thereafter, the $2-\Delta \Delta \mathrm{CT}$ method was used to calculate the relative DNA expression. All primers used in this experiment were synthesized by TsingKe (Beijing, China) and are listed in Table 1.

2.17. Western Blot Analysis. Cells were seeded and cultured as mentioned in Section 2.15. The cells were lysed with RIPA Lysis Buffer (Sigma, Missouri, USA), placed on ice for $30 \mathrm{~min}$, and then centrifuged at $12000 \mathrm{~g}$ for $15 \mathrm{~min}$ at $4^{\circ} \mathrm{C}$, and the supernatant was aspirated. The bicinchoninic acid protein kit (China Biyuntian Biotechnology Co., Ltd) was used to determine the protein concentration of cells. Sodium dodecylsulphate-polyacrylamide gel electrophoresis (SDSPAGE) loading buffer (YongJin, Guangzhou, Guangdong, China) was added to the cells in a $4: 1$ ratio followed by heating at $100^{\circ} \mathrm{C}$ for $10 \mathrm{~min}$. The sample $(20 \mu \mathrm{g})$ was subjected to SDS-PAGE and then transferred to a polyvinylidene difluoride membrane (Millipore, Billerica, MA, USA), which was then blocked with $5 \%$ skim milk at room temperature for
$2 \mathrm{~h}$. The resulting membrane was incubated with the primary antibody at $4^{\circ} \mathrm{C}$ overnight and then with the specific secondary antibody at room temperature for $2 \mathrm{~h}$. Thereafter, ChemiDoc XRS+ (Bio-Rad) was employed to detect the protein band by chemiluminescence, and the images were analyzed using the Quantity One software. The following antibodies were used: CD34 (1:1000; Proteintech, 3C8G12), VEGF (1:1000; Thermo Fisher, MA5-13182), SDF-1 (1:1000; Abcam, ab9797), MMP9 (1:3000; Thermo Fisher, PA5-83748), and GAPDH (1:5000; Fude Biotechnology, FD0063-100).

2.18. Statistical Analysis. Statistical analyses were carried out using SPSS 22.0 software. All experimental data were expressed as the mean difference \pm standard deviation. Comparisons between different groups were conducted using an independent sample $t$-test. Moreover, for multiple specimens, initially, the homogeneity of variance was calculated by Levene's test. If the variance was uniform, one-way analysis of variance (ANOVA) was simultaneously performed using SNK multiple comparisons between groups; if the variance was uneven, Welch's robust ANOVA was conducted, and Dunnett's T3 test was employed for multiple comparisons between groups. $P<0.05$ was considered statistically significant.

\section{Results}

3.1. Features and Structural Characterization of CS/GO Hydrogel Scaffolds. The as-prepared CS/GO hydrogel scaffolds were dark blue, jelly-like, and elastic (as observed by the naked eye), and their internal structure was loose, which recovered after deformation under light pressure (Figures 1(a)-1(d)). After vacuum freeze-drying, their texture became hard and brittle, and the pure CS hydrogel scaffold was not suitable for clamping and had low mechanical strength (Figure 1(e)).

The structure of the pure CS hydrogel scaffold is disordered and irregularly curled. With an increase in the GO concentration, the internal shape of this scaffold slowly became uniform, and the irregular curling gradually improved (Figure 2(a)), indicating that the addition of GO improved the internal structure of the CS hydrogel scaffold. SEM images also show that all the hydrogel scaffolds are highly porous and interconnected, with pore size in the range of 100-150 $\mu \mathrm{m}$ (Figures 2(b) and 2(c)).

We used a series of experiments to further characterize the physical and chemical properties of the CS/GO hydrogel scaffolds. FTIR spectra of the CS hydrogel scaffold exhibited two characteristic absorption bands at 1636 and $1597 \mathrm{~cm}-1$, 


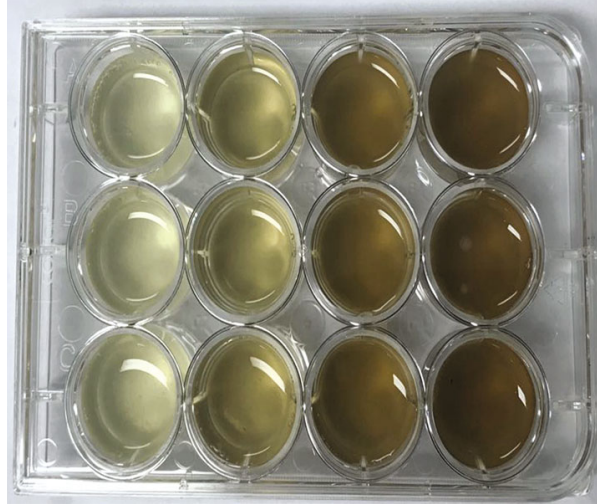

(a)

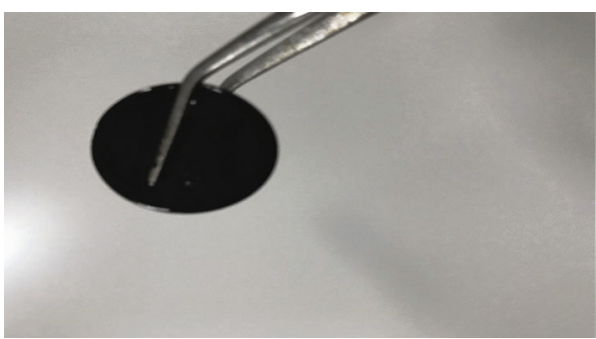

(c)

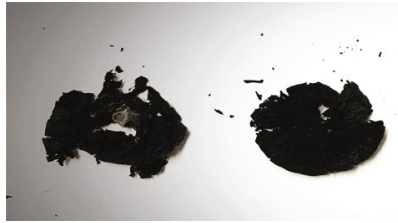

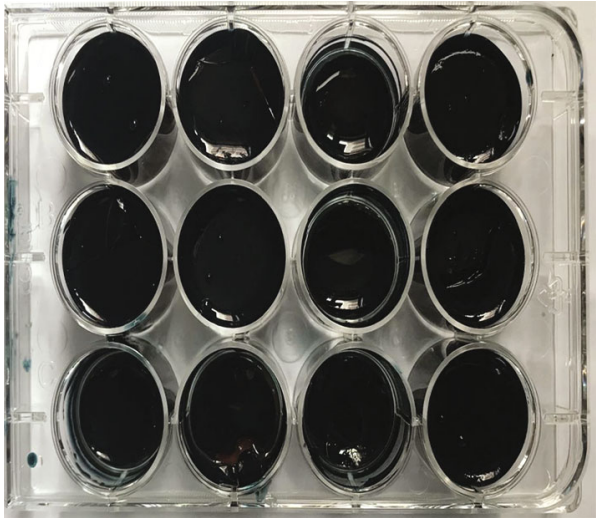

(b)

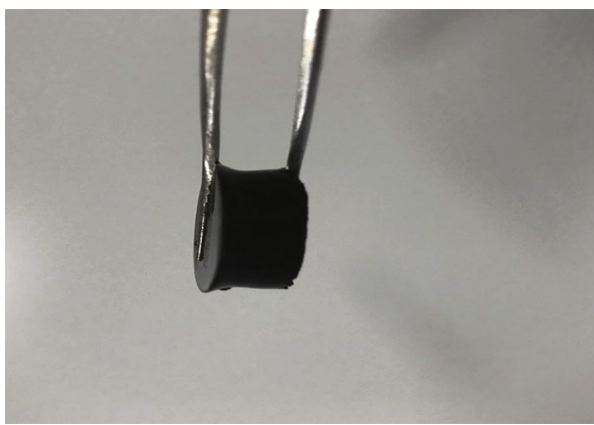

(d)

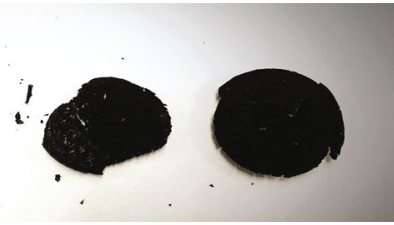

(e)

Figure 1: Features of CS/GO hydrogel scaffolds (from left to right: the hydrogel scaffolds with 0, 0.1, 0.5, and 1.0 wt.\%GO): (a) CS/GO hydrogel scaffolds directly spread on a 12-well plate. (b) CS/GO hydrogel scaffolds crosslinked for $24 \mathrm{~h}$. (c) Front view of the hydrogel scaffolds. (d) Side view of the hydrogel scaffolds. (e) CS/GO hydrogel scaffolds after vacuum freeze-drying.

corresponding to the $\mathrm{C}-\mathrm{O}$ tensile vibration of the acetylated amino group (-NHCO-) and the $\mathrm{N}-\mathrm{H}$ bending of $-\mathrm{NH}_{2}$, respectively. In the case of the CS/GO hydrogel scaffolds, the $-\mathrm{NH}_{2}$ absorption band shifted to a lower value, whereas the intensity of the -NHCO- band increased; this may be because the $-\mathrm{NH}_{2}$ group of $\mathrm{CS}$ reacts with the $-\mathrm{COOH}$ group of $\mathrm{GO}$, leading to the formation of an -NHCO-graft point (Figure 3(a)).

XRD patterns of each group of scaffolds showed the characteristic peak of CS at $2 \theta=20.1^{\circ}$, which indicated the crystallinity of CS (Figure 3(b)). With an increase in the GO concentration, the intensity of this peak gradually decreased, and a new sharp peak appeared near $2 \theta=11.0^{\circ}$, which became more evident with an increase in the GO concentration. All these results suggest that the introduction of GO indeed changes the crystal structure of CS and is consistent with the FTIR results.

\subsection{Physical and Chemical Properties of the CS/GO Hydrogel} Scaffolds. The porosity test indicated that the porosity of each group of scaffolds was ranged from $92 \%$ to $95 \%$. However, it slowly increased with an increase in the concentration of GO from 0.1 to 1.0 wt.\% (Figure 3(c)). There are no differences in porosity among each group $(P=0.095>0.05)$.

As shown in Figure 3(c), the CS hydrogel scaffold has a higher SR than that of the CS/GO hydrogel scaffolds, and the SR of the CS/GO hydrogel scaffolds gradually decreased with an increase in the GO concentration from 0.1 to 1.0 wt. $\%$.

To further explore the influence of GO on the properties of the CS hydrogel scaffold, the tensile properties of the $\mathrm{CS} / \mathrm{GO}$ hydrogel scaffolds were investigated. The tensile stress-strain curves for each group of scaffolds are shown in Figure 3(e), and the mechanical properties are summarized in Table 2. Results suggest that when the GO concentration was increased from 0 to $1.0 \mathrm{wt}$.\%, the elastic modulus, tensile strength, and elongation at break increased from 0.829 to $22.026 \mathrm{MPa}$, from 1.363 to $7.153 \mathrm{MPa}$, and from 61.3 to $161.5 \%$, respectively.

3.3. Identification of EPCs. To verify the hypothesis that the $\mathrm{CS} / \mathrm{GO}$ hydrogel scaffold can improve the angiogenesis of 

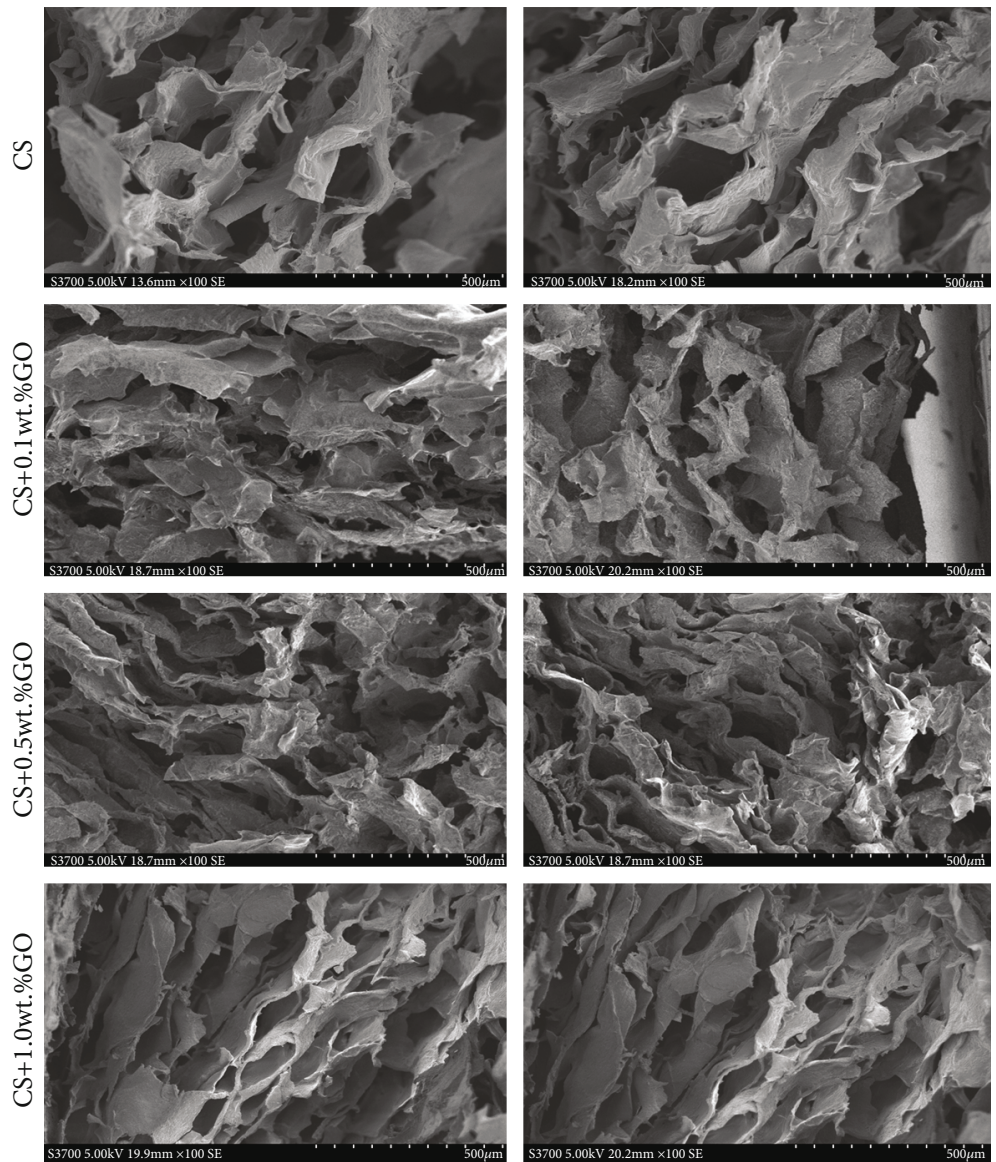

(a)

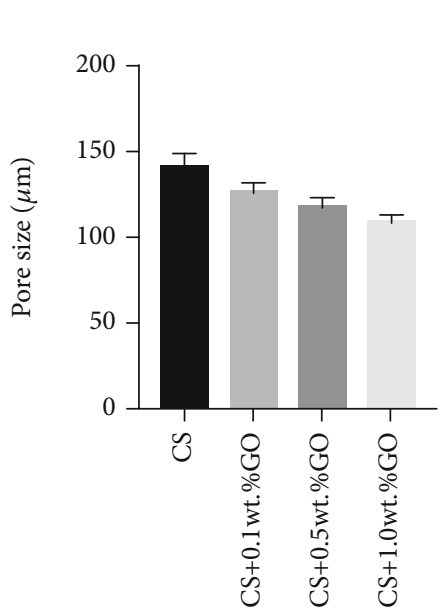

\begin{tabular}{|l|c|}
\hline Content of GO $(w \mathrm{w})$ & Pore size $(\mu \mathrm{m})$ \\
\hline 0 & $140.39 \pm 8.24$ \\
\hline $0.1 \%$ & $125.87 \pm 6.11$ \\
\hline $0.5 \%$ & $117.14 \pm 5.75$ \\
\hline $1.0 \%$ & $108.45 \pm 4.16$ \\
\hline F & 38.125 \\
\hline P & 0.000 \\
\hline
\end{tabular}

(b)

(c)

FIGURE 2: SEM images of the CS/GO hydrogel scaffolds. (a) SEM image of the cross-section of the scaffolds of each group (bar $=500 \mu \mathrm{m}$ ). (b, c) Aperture size of each group randomly measured by Image $.{ }^{*} P<0.05$ compared with the cases of other groups.

cells, we used EPCs from human umbilical cord blood as seed cells for investigation. The freshly isolated primary cells were round (Figure 4(a)). After being cultured for 3-5 days, the cells proliferated, grew into nearly spindle-shaped cells, and demonstrated "colony-like growth" (Figure 4(b)). After 7 days, the cells rapidly proliferated and were connected to each other in a typical "paving stone" pattern (Figure $4(\mathrm{c})$ ). Flow cytometry results showed that the third-generation
EPCs had low expressions of CD45 (0.11\%), CD14 (0.21\%), CD34 (4.21\%), and CD133 (1.16\%) and high expressions of the endothelial cell markers CD31 (99.76\%), CD105 (98.45\%), and KDR (92.55\%) (Figure 4(d)). The results of the tubule formation experiments suggested that the cells connected end-to-end during incubation with Matrigel to form a tubule-like structure, and each tubule was connected to form a tube-like structure similar to that of the vascular 


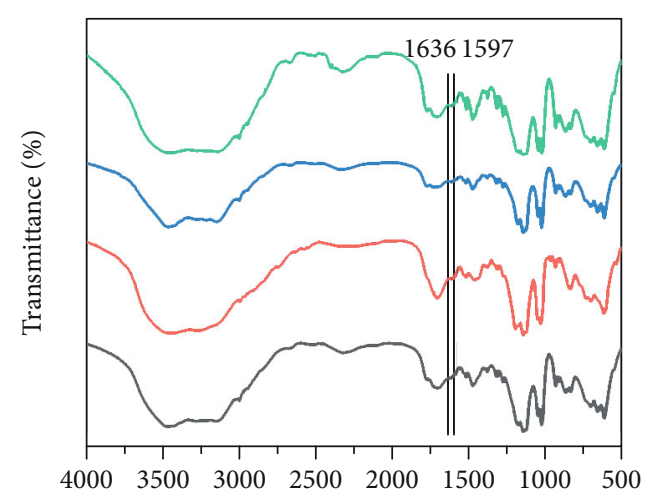

Wavenumber $\left(\mathrm{cm}^{-1}\right)$

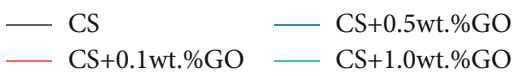

(a)

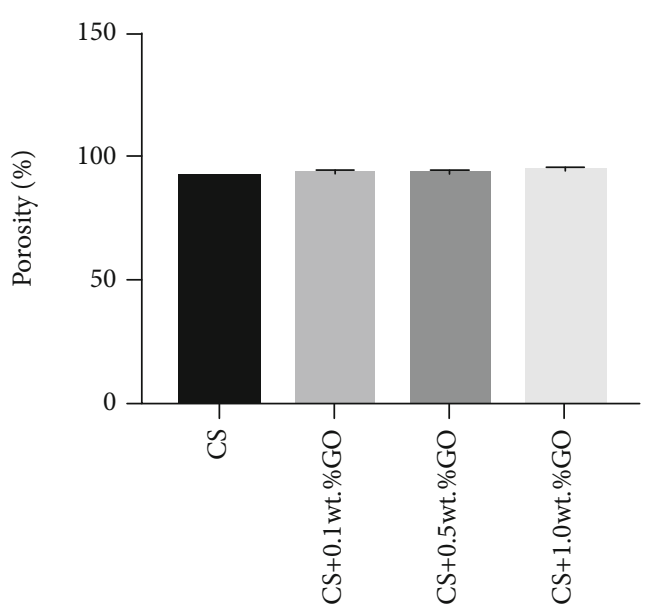

(c)

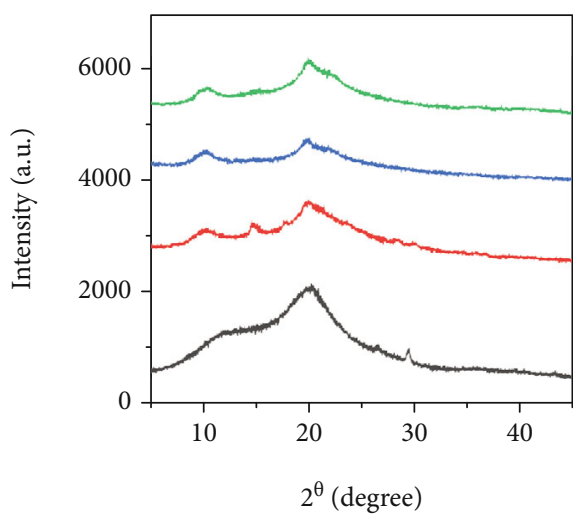

- CS

- $\mathrm{CS}+0.1 \mathrm{wt} . \% \mathrm{GO}$

- CS+0.5wt. $\% \mathrm{GO}$

- CS+1.0wt.\%GO

(b)

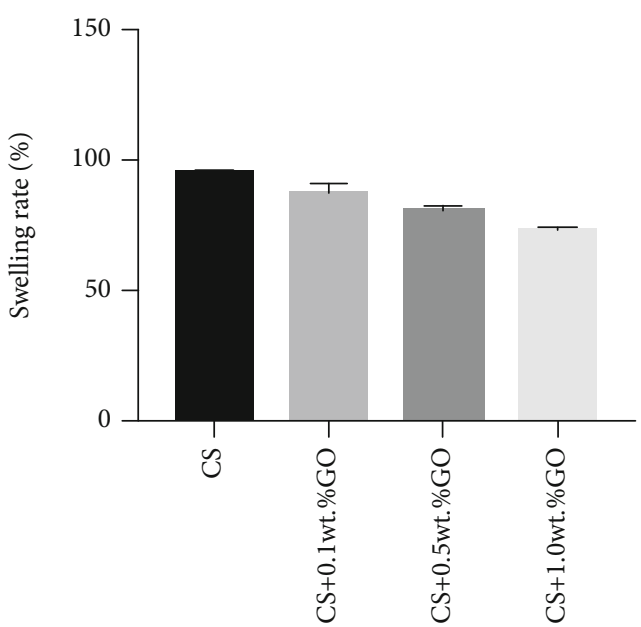

(d)

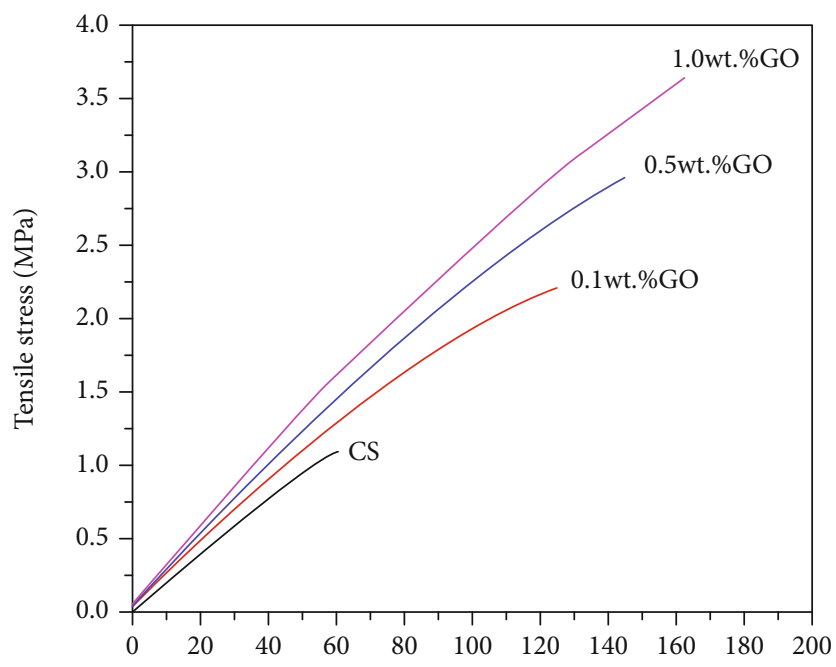

(e)

FIgURE 3: Physical and chemical properties of CS/GO hydrogel scaffolds. (a) FTIR spectra, (b) XRD patterns, and (c) porosity of the hydrogel scaffolds of each group $\left({ }^{*} P>0.05\right)$. (d) Swelling rate of the hydrogel scaffolds of each group $\left({ }^{*} P<0.05\right)$. (e) Tensile properties of the hydrogel scaffolds of each group. 
TABLE 2: Physical properties of the CS/GO hydrogel scaffolds with different contents of GO.

\begin{tabular}{lccc}
\hline Group & Tensile strength (MPa) & Modulus of elasticity (MPa) & Elongation at break (\%) \\
\hline CS & $1.363 \pm 0.367$ & $0.829 \pm 0.590$ & 61.3 \\
CS+0.1 wt.\%GO & $4.053 \pm 0.526$ & $6.863 \pm 0.940$ & 122.3 \\
CS+0.5 wt.\%GO & $5.627 \pm 0.343$ & $12.460 \pm 2.120$ & 140.6 \\
CS+1.0 wt.\%GO & $7.153 \pm 0.383$ & $22.026 \pm 2.752$ & 161.5 \\
\hline
\end{tabular}

lumen (Figure 4(e)). These results confirm that the EPCs extracted in this experiment are a class of progenitor cells that can differentiate into endothelial cells and have the ability to form tubules.

\subsection{Evaluation of the Effect of CS/GO Hydrogel Scaffolds on} the Proliferation of EPCs and Cytotoxicity of These Scaffolds. During the coculture of the hydrogel scaffolds with EPCs, we explored the effect of the CS/GO hydrogel scaffolds on the proliferation of EPCs and the cytotoxicity of these scaffolds (Figures 5(a) and 5(b)). First, the proliferation of the EPCs in the five culture groups was analyzed by the CCK-8 assay on 1, 3, and 5 days after culture. Cell proliferation was considerably inhibited when the concentration of GO was increased to $1.0 \mathrm{wt} . \%$, whereas it was promoted when the concentration of GO was increased from 0 to $0.5 \mathrm{wt} . \%$. The LDH toxicity assay again confirmed that the group containing $1.0 \mathrm{wt} . \% \mathrm{GO}$ indeed had clear cytotoxicity, which indicated that GO over a certain concentration caused cytotoxicity.

To further confirm the cytotoxicity of the hydrogel scaffold with $1.0 \mathrm{wt} . \% \mathrm{GO}$, a live/dead staining was performed, and images were obtained using the fluorescence microscope, and the numbers of dead and live cells were recorded by flow cytometry (Figure 6). The proportion of dead cells in the EPCs, CS; EPCs, CS/0.1 wt.\%GO; EPCs, CS/0.5 wt.\%GO; EPCs, CS/1.0 wt.\%GO; and EPC groups was less than $10 \%$ $(P<0.05$, compared with the case of the EPCs, $\mathrm{CS} / 1.0 \mathrm{wt}$ \% GO group), and there was no statistical difference in the proportion of dead cells between the EPCs; EPCs, CS; EPCs, CS/0.1 wt.\%GO; and EPCs, CS/0.5 wt.\%GO groups $(P>0.05)$. In the EPCs, CS/1.0 wt.\%GO group, the proportion of dead cells was up to $16.5 \%(P<0.05$, compared with the cases of other groups). All these results clearly confirm that the hydrogel scaffold with $1.0 \mathrm{wt} . \% \mathrm{GO}$ has substantial cytotoxicity.

3.5. Effect of the CS/GO Hydrogel Scaffold on the Angiogenesis of EPCs. Results of the tube formation analysis (Figures 5(c)5(f)) demonstrated that on the seventh day of coculture, the EPCs in the EPCs, CS/0.5 wt.\%GO group had most significant tube formation ability, whereas those in the EPCs, $\mathrm{CS} / 1.0 \mathrm{wt} . \% \mathrm{GO}$ group had the lowest tube formation ability $(P<0.05)$. Moreover, the tube formation abilities of EPCs in the EPCs and EPCs, CS groups were not significant $\left({ }^{\Delta} P\right.$ $>0.05$ ).

To further study the role of CS/GO hydrogel scaffolds in promoting angiogenesis, qRT-PCR and Western blot analysis were conducted, and the results showed that on the seventh day of coculture, the expressions of CD34, VEGF,
MMP9, and SDF-1 in EPCs were considerably upregulated with an increase in the GO concentration when compared with the case of the EPC group. However, the expressions of CD34, VEGF, MMP9, and SDF-1 were inhibited in the EPCs, CS/1.0 wt.\%GO group (Figure 7). As shown in Figure 7(a), the relative mRNA expressions of the EPCs, CS and EPC groups were not significant $(P>0.05)$, whereas those of the other groups had statistical significance in multiple comparisons $\left({ }^{*} P<0.05\right.$, compared with that of the EPC group). The results of Western blot analysis are similar to those of the qRT-PCR (Figures 7(b) and 7(c)). All these results indicate that in an appropriate concentration range, $\mathrm{GO}$ can promote the proliferation and angiogenic ability of EPCs, which will contribute to improving angiogenesis in tissue engineering.

\section{Discussion}

In 1993, tissue engineering was proposed by American scholars and subsequently brought a new dawn to the reconstruction and repair of tissue defects [39]. Tissue engineering is a discipline that covers multidisciplinary fields such as biology, medicine, and engineering. It develops new biological materials to develop biological substitutes that can repair or improve defective tissues, organs, or parts of the human body $[40,41]$. Although the existing biomaterials have made some progress in the repair of tissue defects, the angiogenesis of regenerated tissue is still a research problem. Although currently different studies promote tissue regeneration through angiogenesis, none of the methods have been successfully applied clinically $[5,8,42]$. Therefore, continuous exploration and development of scaffold materials that promote tissue angiogenesis and the success rate of tissue defect repair have important medical value.

GO was brought into tissue engineering for its good mechanical properties and angiogenesis properties [43, 44]. So, we added GO into chitosan hydrogel in order to make an ideal hydrogel scaffold material. A single biomaterial has limitations due to its own performance. Therefore, the composite materials have their superior performance due to multiple materials being mutually reconstructed and will play a vital role in tissue engineering scaffolds to repair tissue defects. The application of crosslinking and covalent bonding significantly improves the stability of the hydrogel $[45,46]$. So, genipin was used as the crosslinking agent in this study. Accordingly, a series of CS/GO hydrogel scaffolds were fabricated by crosslinking CS and GO at different concentrations using GNP in our research.

Analyzing the features and structural characterization of CS/GO hydrogel scaffolds, we attained the conclusions as 


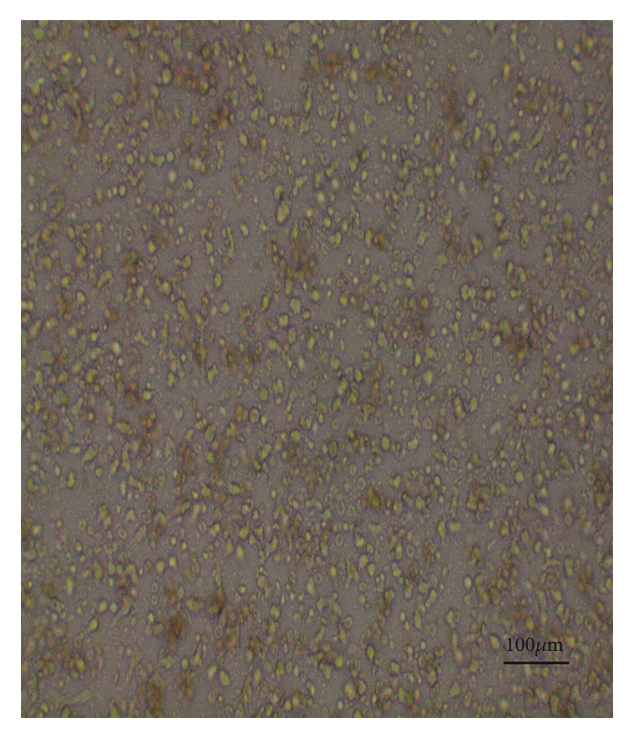

(a)

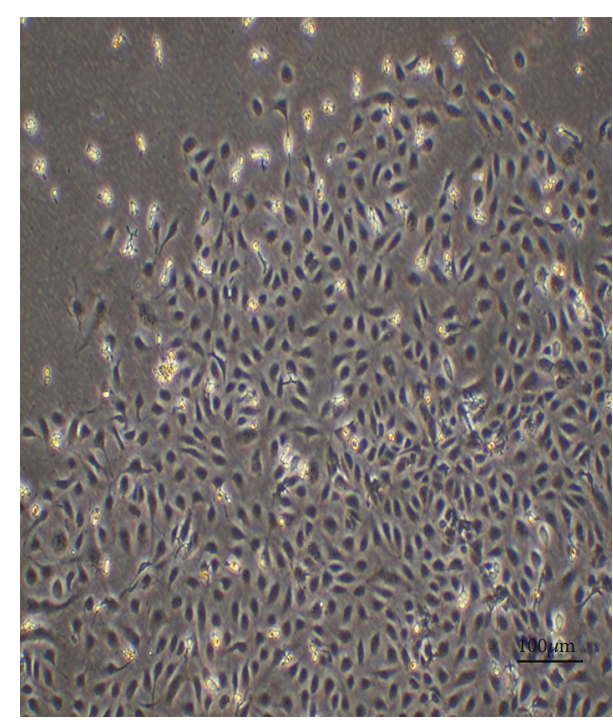

(b)

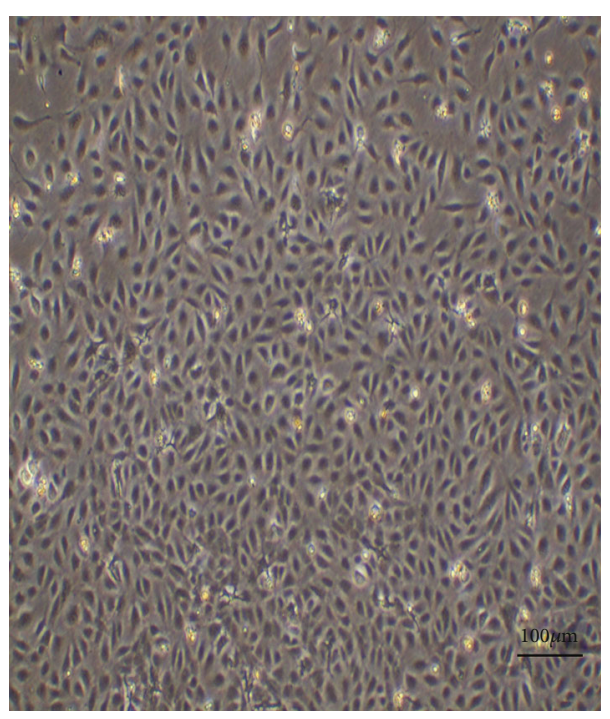

(c)

FIgure 4: Continued. 

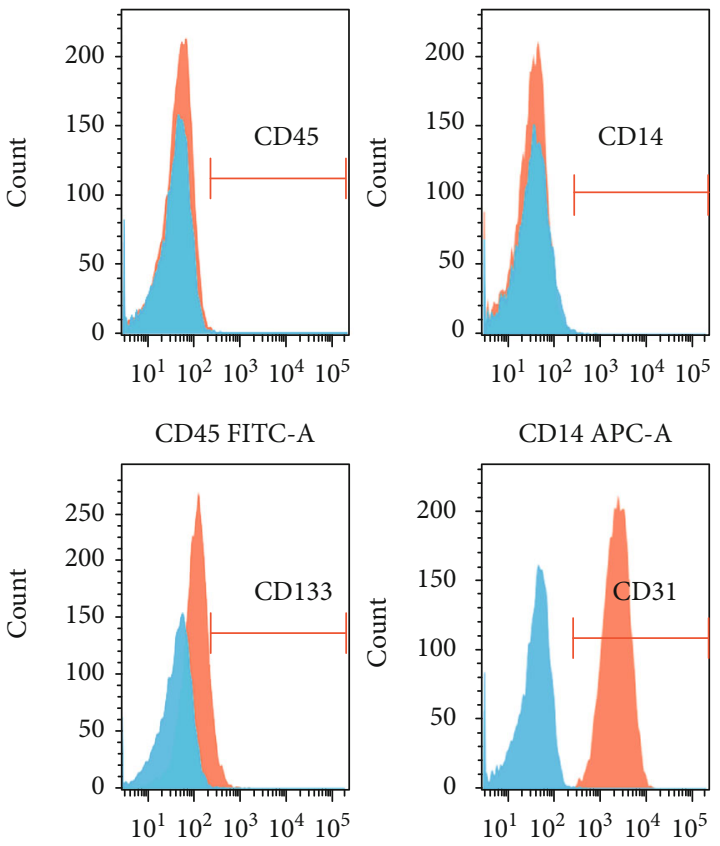

CD133 PE-A

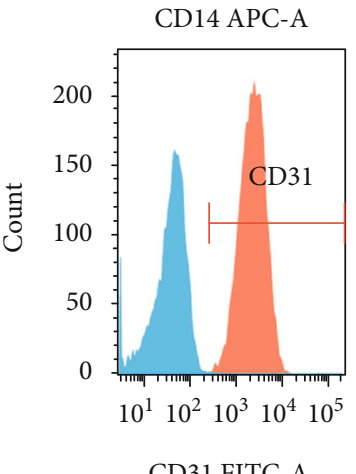

CD31 FITC-A

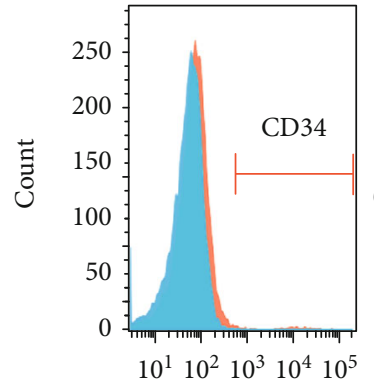

CD34 PE-A

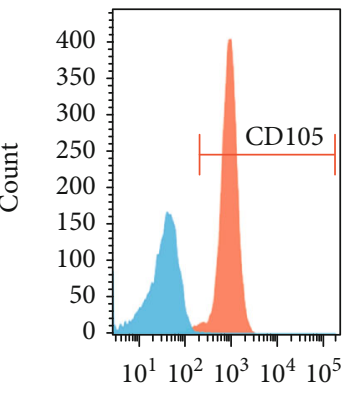

CD105 FITC-A

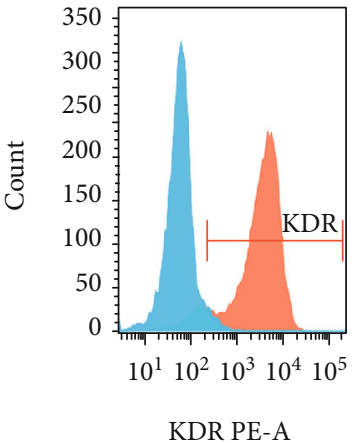

KDR PE-A

(d)

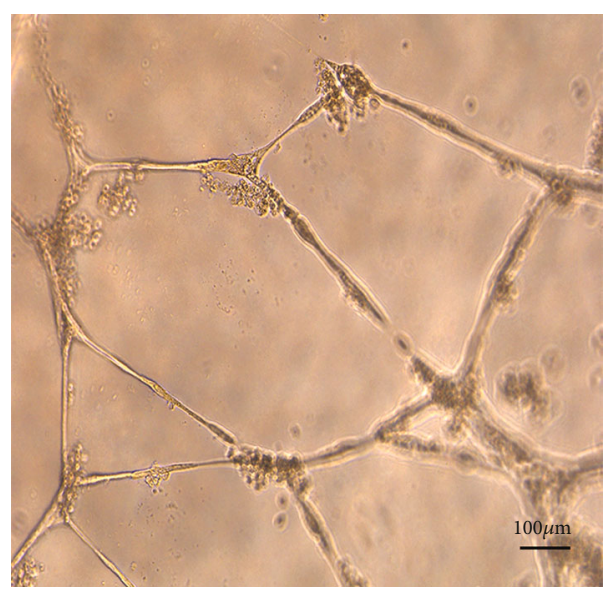

(e)

FIGURE 4: Isolation, culture, and identification of EPCs (provided by Guangdong Cord Blood Bank). Newly isolated primary cells (a) and cells cultured for 3-5 (b) and 7 (c) days. (d) Flow cytometry identification results of primary cells. (e) Matrigel tubule formation experiment of primary cells.

follows. With an increase in the GO concentration, the strength of the scaffold gradually increased. Therefore, the addition of GO to the CS hydrogel scaffold is effective in increasing the hardness of this scaffold. Furthermore, the pore size of the scaffold slowly decreased with an increase in the concentration of GO. This further showed that the addition of GO improved the structure of the CS hydrogel scaffold, making the spatial network structure inside the scaffold more uniform and porous.

We used a series of experiments to further characterize the physical and chemical properties of the CS/GO hydrogel scaffolds. From the FTIR and XRD, the introduction of GO indeed changed the crystal structure of CS. We predicted a possible schematic diagram of the material structure in the Figure 8.

The porosity test simply confirms that the introduction of GO increases the porosity of the CS hydrogel scaffold. Furthermore, a better porosity can promote cell spreading and facilitate cell proliferation $[47,48]$. In addition, SEM images show that the introduction of GO makes the structure of the CS hydrogel scaffold more uniform, reduces the pore size, and increases the porosity. Considering that the porosity increased and the structure of the hydrogel became more uniform by adding GO, it is attributed to physical crosslinking between - $\mathrm{COOH}$ and $-\mathrm{OH}$ of $\mathrm{GO}$ and $-\mathrm{NH}_{2}$ of $\mathrm{CS}$, which may even chemically crosslink upon the addition of GNP. 

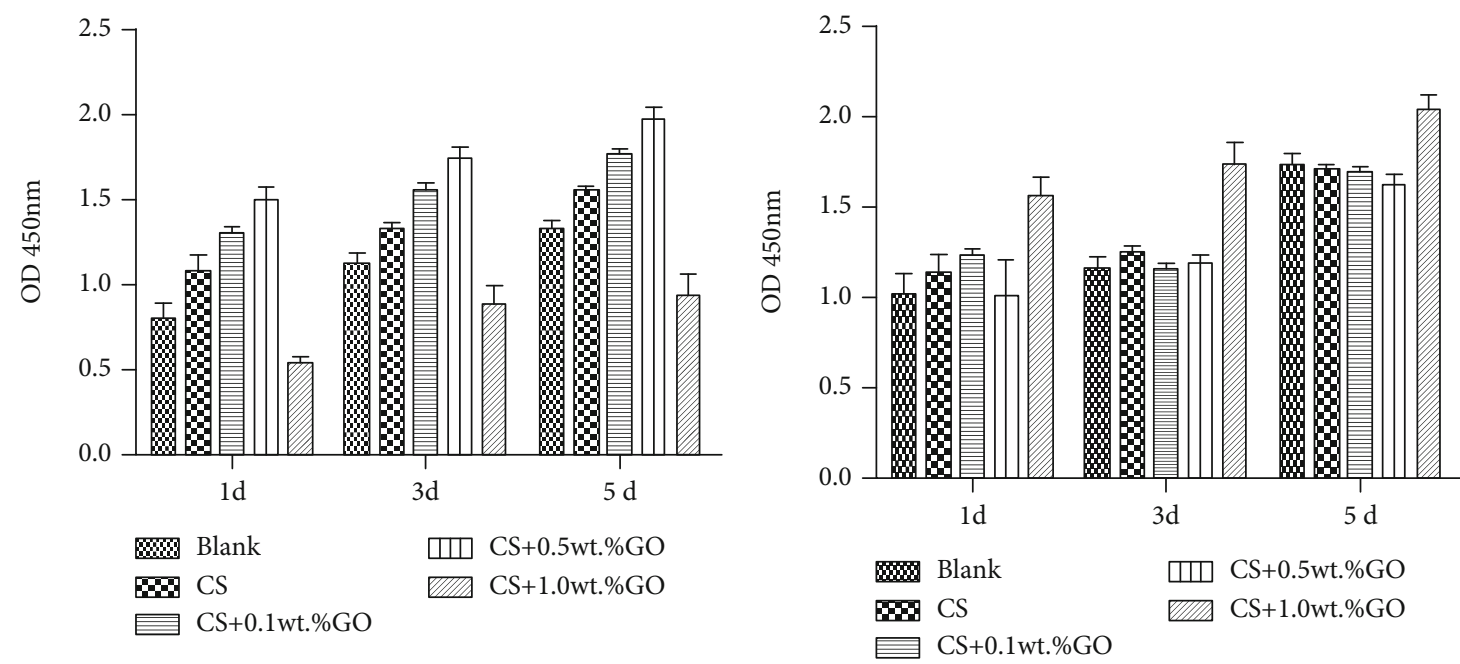

(a)

(b)

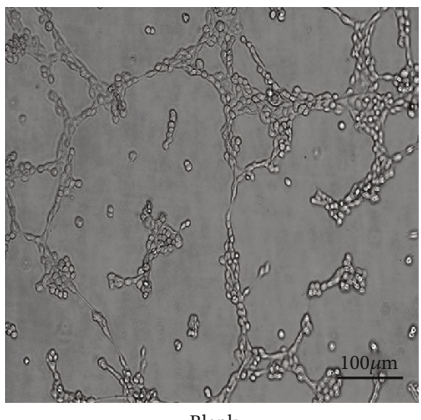

Blank

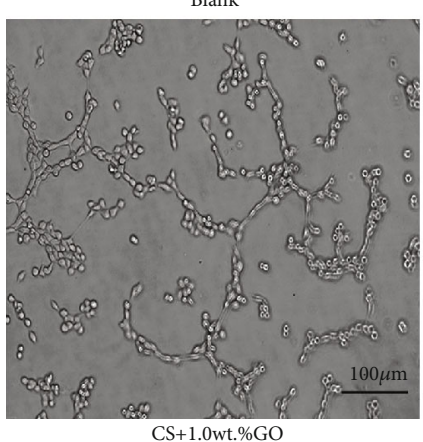

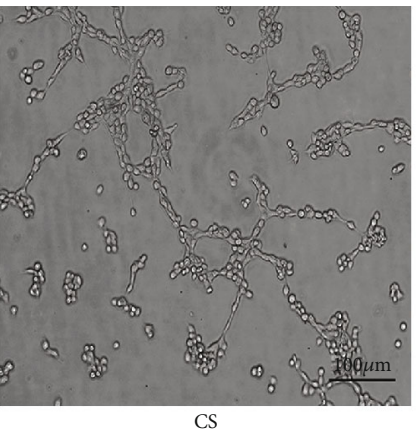

CS

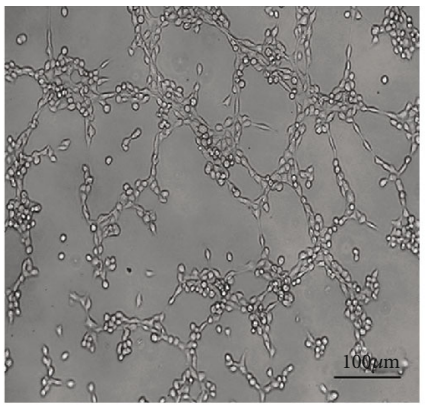

$\mathrm{CS}+0.1 \mathrm{wt} . \% \mathrm{GO}$

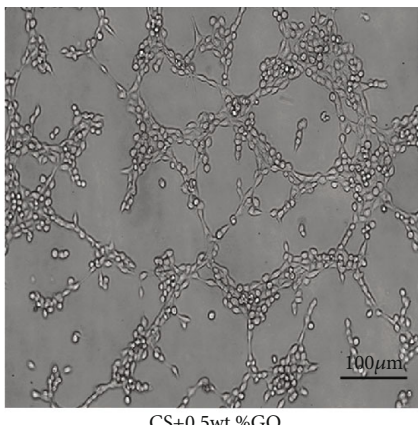

(c)

FIgure 5: Continued. 


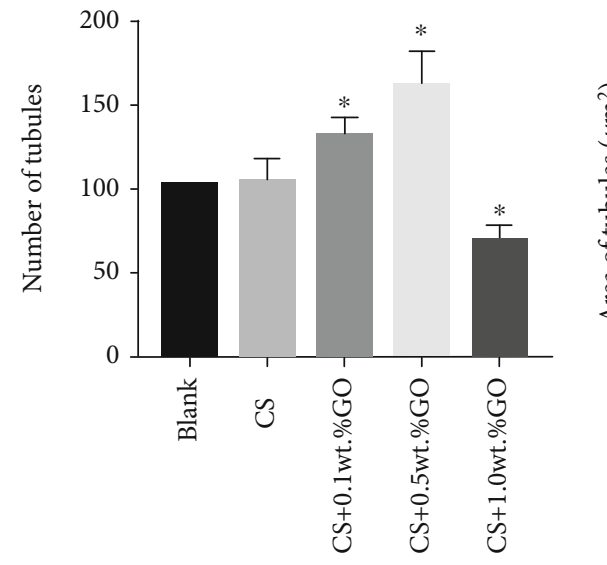

(d)

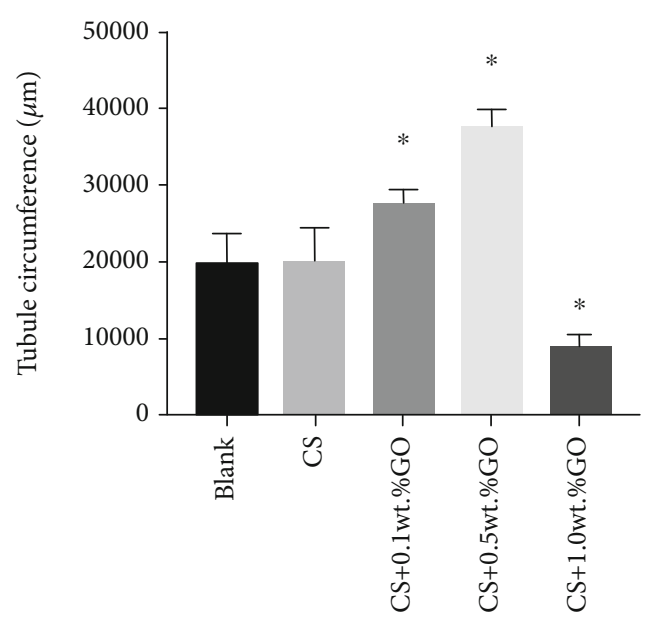

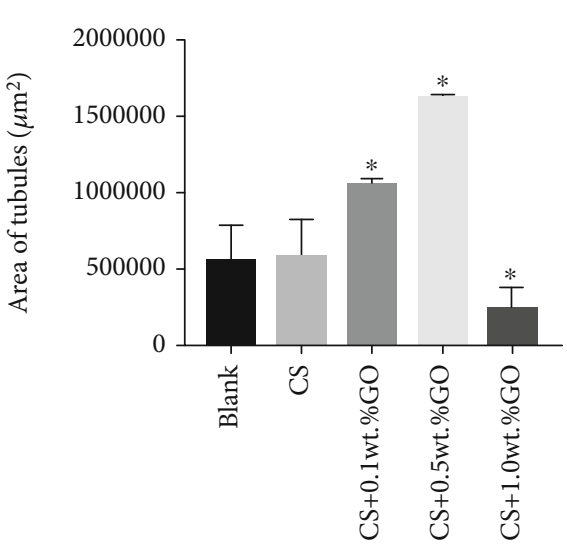

(e)

(f)

FIGURE 5: Effect of CS/GO hydrogel scaffolds on the proliferation and angiogenesis of EPCs. (a) Proliferation level of cells in each group determined by the CCK-8 assay. (b) Cytotoxicity of hydrogel scaffolds in each group evaluated by the LDH assay. (c-f) Angiogenesis ability of cells in each group determined using tube formation. ${ }^{*} P<0.05$.

Hence, we predict that the cells can expand and grow better in hydrogel scaffold materials, which would facilitate the exchange of substances between the implanted body and the outside environment for providing more energy to promote the formation of blood vessels and tissues. All these results suggest that the $\mathrm{CS} / \mathrm{GO}$ hydrogel scaffold may become a potential scaffold for tissue engineering.

This implied that the presence of GO enhanced the swelling rate of the CS hydrogel scaffold possibly owing to physical crosslinking between $-\mathrm{COOH}$ and $-\mathrm{OH}$ of $\mathrm{GO}$ and $-\mathrm{NH}_{2}$ of $\mathrm{CS}$, which may even chemically crosslink upon the addition of GNP; however, this requires further confirmation. Thus, it can be speculated that crosslinking inhibits the swelling of the CS hydrogel scaffold and thereby reduces the SR.

Therefore, the addition of GO significantly improved the tensile properties of the CS hydrogel scaffold. This is consistent with the previously reported results of CS/GO hydrogel films with different GO concentrations $[49,50]$. In summary, we found that the addition of GO changed the structure of the chitosan hydrogel, which affected the physical and chemical properties of the material.
To further explore the biocompatibility and angiogenic effect of chitosan/graphene oxide hydrogel scaffolds, the EPCs were chosen. ECs (endothelial cells) cannot be widely used for the vascularization of bone tissue because of their low proliferation and low utilization [51]. Therefore, we used EPCs as the seed cells. The CCK- 8 and cytotoxicity assay results showed that when the concentration of GO was higher than $0.5 \mathrm{wt} . \%$, it inhibited the proliferation of EPCs and caused significant cytotoxicity to the cells. This is consistent with the results reported by Wang et al.; that is, GO has no considerable cytotoxicity at a suitable concentration, whereas it exhibits substantial cytotoxicity at doses higher than $50 \mu \mathrm{g} / \mathrm{mL}$ [52]. Therefore, as a new biomaterial, GO at an appropriate concentration can be used to prepare tissue engineering scaffold materials.

During the tube formation experiment, the CS hydrogel scaffold did not significantly promote angiogenesis, whereas after the addition of GO, it stimulated tube formation. However, when the concentration of GO was up to $1.0 \mathrm{wt} . \%$, tube formation was inhibited. This proves that high concentrations of GO may inhibit angiogenesis of the EPCs. To further explore the molecular mechanism of blood vessel formation 

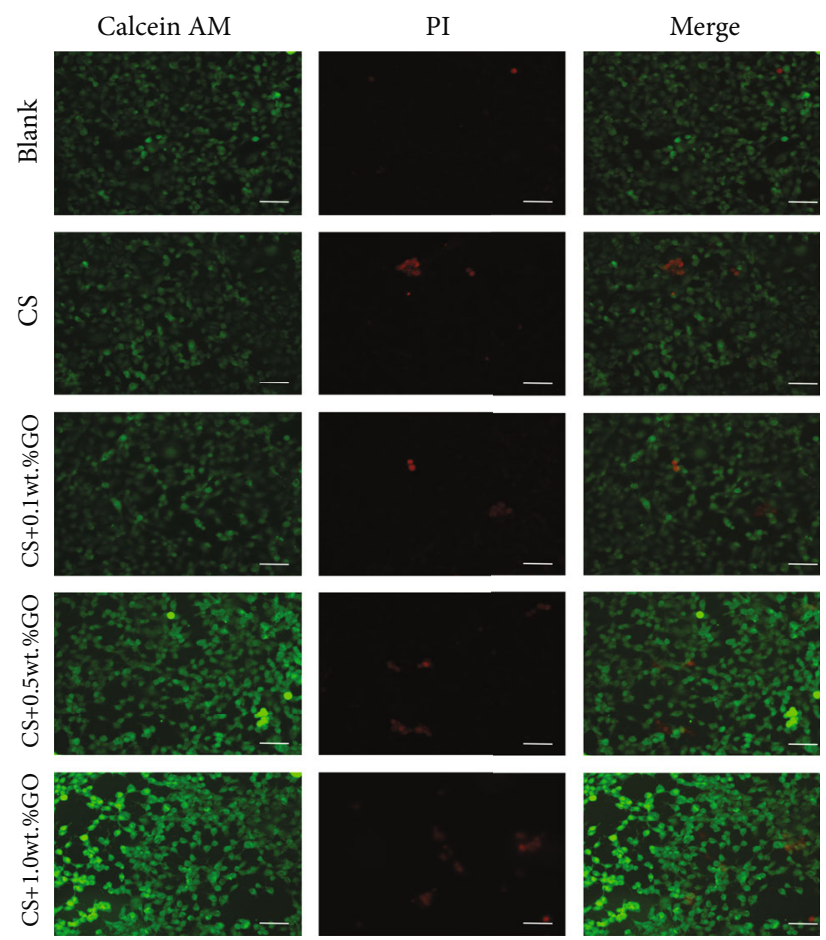

(a)
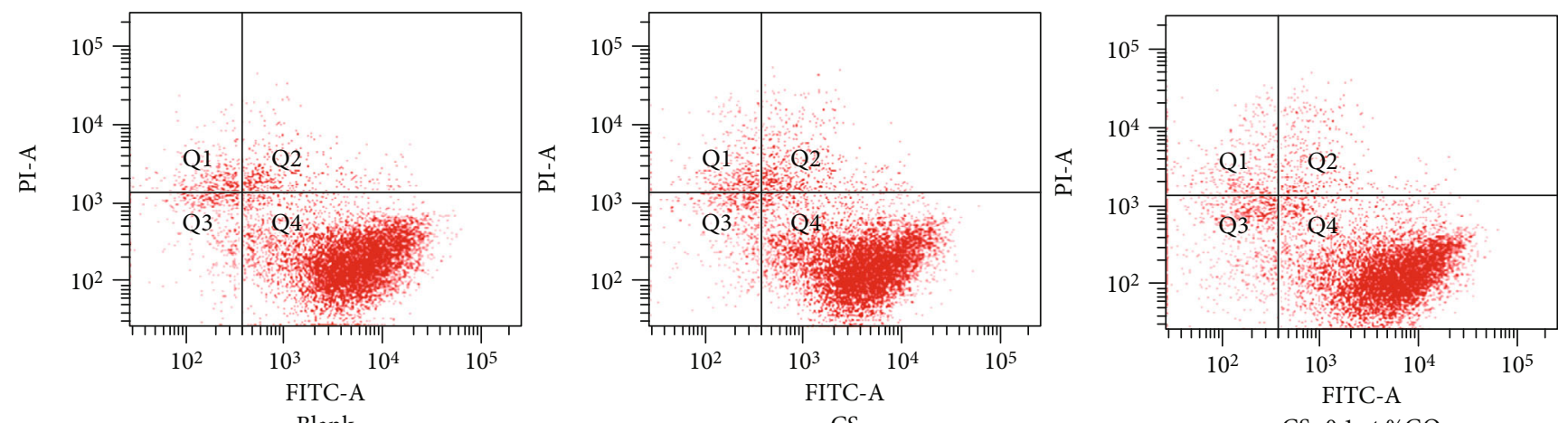

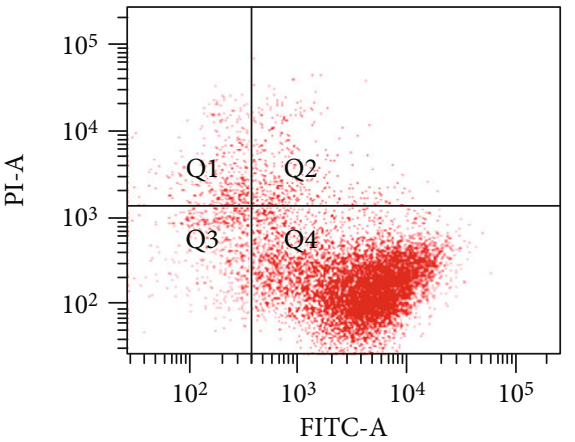

FITC-A

$\mathrm{CS}+0.5 \mathrm{wt} . \% \mathrm{GO}$

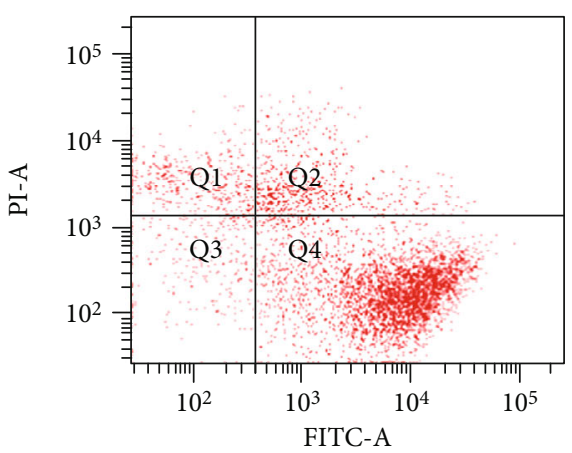

CS+1.0wt.\%GO

(b)

Figure 6: Continued. 


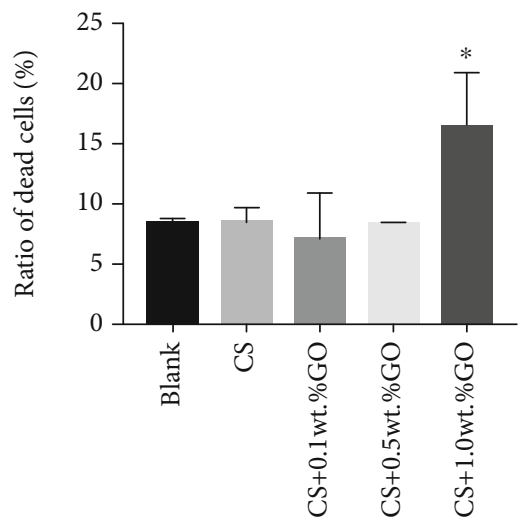

(c)

FIGURE 6: Dead and live cell staining of the EPCs in each coculture group: (a) staining images obtained using the fluorescence microscope; (b) dead and live cell staining results acquired by FCM; (c) ratio of dead cells in each group. ${ }^{*} P<0.05$.

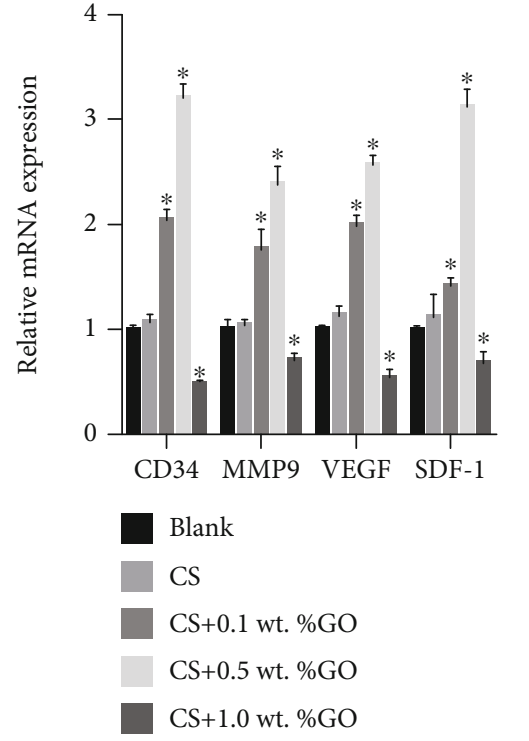

(a)

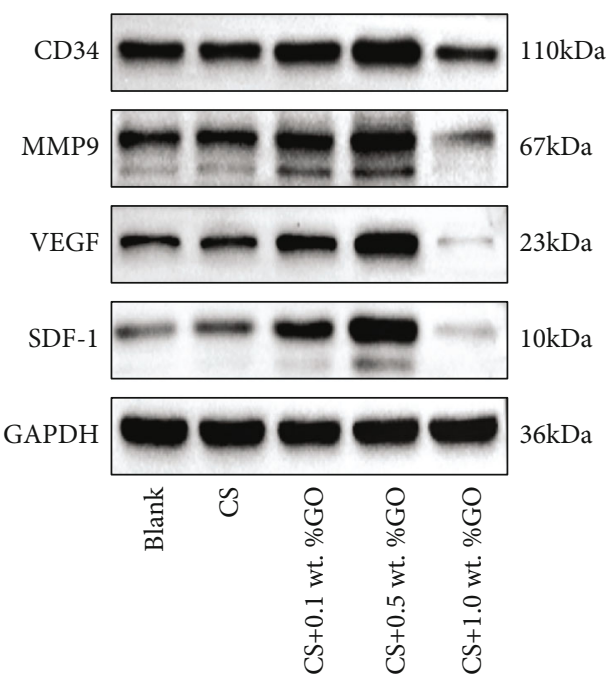

(b)
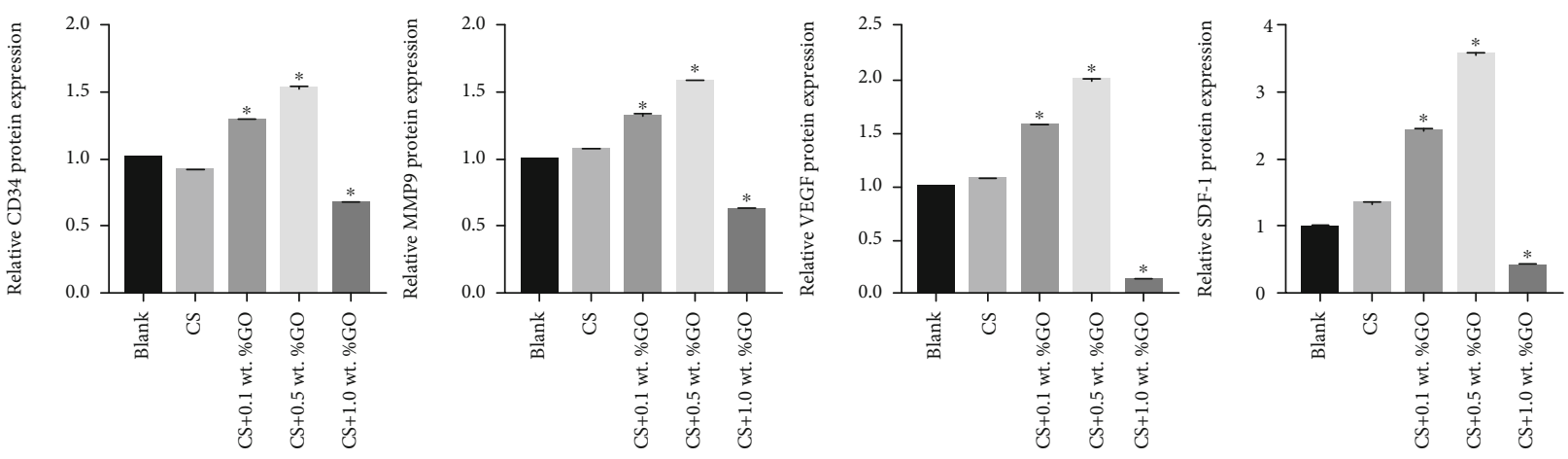

(c)

Figure 7: (a) qRT-PCR analysis of the CD34, VEGF, MMP9, and SDF-1 expressions in each group. (b) Western blot analysis results of CD34, VEGF, MMP9, and SDF-1 protein expressions in each group. ${ }^{*} P<0.05$ compared with the blank group.

by the CS/GO hydrogel scaffolds, we conducted more experiments. CD34, VEGF, MMP9, and SDF-1 were chosen to explore the mechanism.
CD34 is a transmembrane glycoprotein that is selectively expressed on the surface of EPCs. SDF-1 and VEGF play a key role in EPC function. Studies have shown that SDF-1 


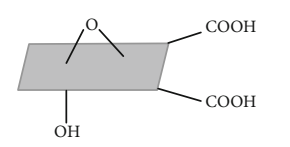

Graphene oxide (GO)

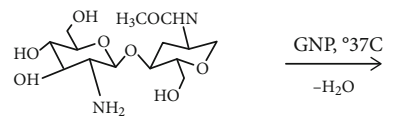

Chitosan (CS)

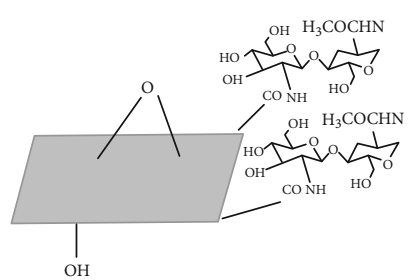

GO-CO-NH-CS

Figure 8

promotes the expression of VEGF in ECs and is an important cytokine to mobilize EPC [53]. Wang et al. confirmed that metallothionein (MT) promotes the angiogenesis of EPCs mediated by the HIF- $1 \alpha /$ SDF-1/VEGF pathway [54]. Several studies proved that the SDF-1/VEGF pathway is vital to the angiogenesis of EPCs $[55,56]$. In our research, the expressions of the angiogenesis-related genes CD34, VEGF, MMP9, and SDF-1 were examined and found to be upregulated at an appropriate concentration of GO. Western blot analysis results also confirmed this phenomenon. Thus, we concluded that GO may regulate the angiogenic ability of EPC through the SDF-1/VEGF signaling pathway. We found few studies about the angiogenic mechanism of GO on EPCs, while different from ECs. Mukherjee et al. [26] found that the activation of phospho-eNOS and phospho-Akt might be the plausible mechanisms for GO- and rGO-induced angiogenesis of HUVECs. From the research, we concluded that the immune microenvironment induced by GO at an appropriate concentration promotes the angiogenesis of HUVEC through the VEGF pathway [57]. However, further investigation is required to understand the corresponding specific signaling molecular mechanism.

We predict that the suitable concentration of $\mathrm{CS} / \mathrm{GO}$ hydrogel scaffolds can promote the proliferation and tube formation of EPCs and further stimulate the formation of blood vessels. The long-term prospect of this research is providing little evidence for the role of CS/GO hydrogel scaffolds combined with EPCs in promoting angiogenesis and the repair of tissue defects. We hope that these findings can provide a new theoretical basis for the application of the combination of CS/GO hydrogel scaffolds and EPCs in promoting tissue engineering repair and in tissue regenerative medicine. However, our research has several limitations. First, we did not comprehensively study the specific mechanism of CS and GO crosslinking by GNP. Second, only a few detailed studies were conducted on the molecular mechanism of EPC regulation by the CS/GO hydrogel scaffold. Third, as in vivo experiments were not performed, we cannot verify the role of this scaffold material in repairing bone defects in vivo.

\section{Conclusion}

In this study, we successfully prepared a series of CS/GO hydrogel scaffolds with different concentrations of GO. The addition of GO improved the structure of the CS hydrogel scaffold, facilitated the formation of the spatial network structure, and improved the physical, chemical, and mechan- ical properties of the scaffold. At an appropriate concentration, GO had no significant cytotoxicity and promoted the extension and proliferation of EPCs, thereby stimulating the formation of blood vessels. However, research on the role of GO in basic and clinical medical fields is still in its infancy, and the molecular mechanism involved in angiogenesis needs further investigation. In conclusion, our study not only opens a new path for the future research of CS/GO hydrogel scaffolds but also provides new ideas for the application of these scaffolds in promoting the formation of new blood vessels.

\section{Data Availability}

Dr. Zhang, e-mail: summere0615@163.com, will provide the research data.

\section{Conflicts of Interest}

The authors declare that they have no conflicts of interest with the contents of this article.

\section{Authors' Contributions}

L.F.Z. contributed to the conception of the study, generated the data, and wrote the manuscript. X.P.L., G.Y.R., and Y.T.P. contributed significantly to the analysis and manuscript preparation. C.Y.S. provided the EPCs for this study. Y.H. provided comments and suggestions for submission. S.G.Z. revised it critically for important intellectual content and approved the final version. Lifang Zhang and Xinping $\mathrm{Li}$ are co-first authors of this work.

\section{Acknowledgments}

The authors acknowledge support from the Guangzhou Municipal Science and Technology Project (Grant No. 201707010193) and the Intra-hospital Fund of Southern Medical University of Stomatological Hospital (PY2020022).

\section{References}

[1] B. Bakhshandeh, P. Zarrintaj, M. O. Oftadeh et al., "Tissue engineering; strategies, tissues, and biomaterials," Biotechnology \& Genetic Engineering Reviews, vol. 33, no. 2, pp. 144172, 2017.

[2] M. Lovett, K. Lee, A. Edwards, and D. L. Kaplan, "Vascularization strategies for tissue engineering," Tissue Engineering. Part B, Reviews, vol. 15, no. 3, pp. 353-370, 2009. 
[3] E. B. Peters, "Endothelial progenitor cells for the vascularization of engineered tissues," Tissue Engineering. Part B, Reviews, vol. 24, no. 1, pp. 1-24, 2018.

[4] K. E. Johnson and T. A. Wilgus, "Vascular endothelial growth factor and angiogenesis in the regulation of cutaneous wound repair," Advances in Wound Care, vol. 3, no. 10, pp. 647-661, 2014.

[5] S. N. Sethu, S. Namashivayam, S. Devendran et al., "Nanoceramics on osteoblast proliferation and differentiation in bone tissue engineering," International Journal of Biological Macromolecules, vol. 98, pp. 67-74, 2017.

[6] H. D. Kim, S. Amirthalingam, S. L. Kim, S. S. Lee, J. Rangasamy, and N. S. Hwang, "Biomimetic materials and fabrication approaches for bone tissue engineering," Advanced healthcare materials, vol. 6, no. 23, 2017.

[7] S. Yin, W. Zhang, Z. Zhang, and X. Jiang, "Recent advances in scaffold design and material for vascularized tissue-engineered bone regeneration," Advanced healthcare materials, vol. 8, no. 10, article e1801433, 2019.

[8] L. Reddy, D. Murugan, M. Mullick, E. T. Begum Moghal, and D. Sen, "Recent approaches for angiogenesis in search of successful tissue engineering and regeneration," Current Stem Cell Research \& Therapy, vol. 15, no. 2, pp. 111-134, 2020.

[9] Y. C. Chiu, M. H. Cheng, H. Engel et al., "The role of pore size on vascularization and tissue remodeling in PEG hydrogels," Biomaterials, vol. 32, no. 26, pp. 6045-6051, 2011.

[10] M. Schleicher, H. P. Wendel, O. Fritze, and U. A. Stock, "In vivo tissue engineering of heart valves: evolution of a novel concept," Regenerative Medicine, vol. 4, no. 4, pp. 613-619, 2009.

[11] Y. Wu, L. Wang, B. Guo, and P. X. Ma, "Injectable biodegradable hydrogels and microgels based on methacrylated poly(ethylene glycol)-co-poly(glycerol sebacate) multi-block copolymers: synthesis, characterization, and cell encapsulation," Journal of materials chemistry. B, vol. 2, no. 23, pp. 3674-3685, 2014.

[12] M. Liu, X. Zeng, C. Ma et al., "Injectable hydrogels for cartilage and bone tissue engineering," Bone research, vol. 5, no. 1, p. 17014, 2017.

[13] J. Ng, K. Spiller, J. Bernhard, and G. Vunjak-Novakovic, "Biomimetic approaches for bone tissue engineering," Tissue Engineering. Part B, Reviews, vol. 23, no. 5, pp. 480-493, 2017.

[14] Z. Shariatinia and A. M. Jalali, "Chitosan-based hydrogels: preparation, properties and applications," International Journal of Biological Macromolecules, vol. 115, pp. 194-220, 2018.

[15] L. J. Del Valle, A. Díaz, and J. Puiggalí, "Hydrogels for biomedical applications: cellulose, chitosan, and protein/peptide derivatives," Gels, vol. 3, no. 3, 2017.

[16] S. Deepthi, J. Venkatesan, S. K. Kim, J. D. Bumgardner, and R. Jayakumar, "An overview of chitin or chitosan/nano ceramic composite scaffolds for bone tissue engineering," International journal of biological macromolecules, vol. 93, no. Part B, pp. 1338-1353, 2016.

[17] J. Venkatesan and S. K. Kim, "Chitosan composites for bone tissue engineering-an overview," Marine Drugs, vol. 8, no. 8, pp. 2252-2266, 2010.

[18] M. L. Zhang, J. Cheng, Y. C. Xiao, R. F. Yin, and X. Feng, "Raloxifene microsphere-embedded collagen/chitosan/ $\beta$-tricalcium phosphate scaffold for effective bone tissue engineering," International Journal of Pharmaceutics, vol. 518, no. 1-2, pp. 80-85, 2017.
[19] M. A. Nazeer, E. Yilgör, and I. Yilgör, "Intercalated chitosan/hydroxyapatite nanocomposites: promising materials for bone tissue engineering applications," Carbohydrate Polymers, vol. 175, pp. 38-46, 2017.

[20] A. Topsakal, M. Uzun, G. Ugar et al., "Development of amoxicillin-loaded electrospun polyurethane/chitosan/ $\$ \backslash$ beta \$ -tricalcium phosphate scaffold for bone tissue regeneration," IEEE Transactions on Nanobioscience, vol. 17, no. 3, pp. 321-328, 2018.

[21] G. R. Owen, M. Dard, and H. Larjava, "Hydoxyapatite/betatricalcium phosphate biphasic ceramics as regenerative material for the repair of complex bone defects," Journal of Biomedical Materials Research. Part B, Applied Biomaterials, vol. 106, no. 6, pp. 2493-2512, 2018.

[22] S. F. Kiew, L. V. Kiew, H. B. Lee, T. Imae, and L. Y. Chung, "Assessing biocompatibility of graphene oxide-based nanocarriers: a review," Journal of controlled release: official journal of the Controlled Release Society, vol. 226, pp. 217-228, 2016.

[23] M. Thangamuthu, K. Y. Hsieh, P. V. Kumar, and G. Y. Chen, "Graphene- and graphene oxide-based nanocomposite platforms for electrochemical biosensing applications," International journal of molecular sciences, vol. 20, no. 12, p. 2975, 2019.

[24] J. Lee, J. Kim, S. Kim, and D. H. Min, "Biosensors based on graphene oxide and its biomedical application," Advanced drug delivery reviews, vol. 105, no. Part B, pp. 275-287, 2016.

[25] L. Zhang, J. Xia, Q. Zhao, L. Liu, and Z. Zhang, "Functional graphene oxide as a nanocarrier for controlled loading and targeted delivery of mixed anticancer drugs," Small, vol. 6, no. 4, pp. 537-544, 2010.

[26] S. Mukherjee, P. Sriram, A. K. Barui et al., "Graphene oxides show angiogenic properties," Advanced Healthcare Materials, vol. 4, no. 11, pp. 1722-1732, 2015.

[27] Y. Chen, Z. Zheng, R. Zhou et al., "Developing a strontiumreleasing graphene oxide-/collagen-based organic-inorganic nanobiocomposite for large bone defect regeneration via MAPK signaling pathway," ACS Applied Materials \& Interfaces, vol. 11, no. 17, pp. 15986-15997, 2019.

[28] S. Chakraborty, T. Ponrasu, S. Chandel, M. Dixit, and V. Muthuvijayan, "Reduced graphene oxide-loaded nanocomposite scaffolds for enhancing angiogenesis in tissue engineering applications," Royal Society open science, vol. 5, no. 5, article 172017, 2018.

[29] E. López-Dolado, A. González-Mayorga, M. C. Gutiérrez, and M. C. Serrano, "Immunomodulatory and angiogenic responses induced by graphene oxide scaffolds in chronic spinal hemisected rats," Biomaterials, vol. 99, pp. 72-81, 2016.

[30] T. Asahara, T. Murohara, A. Sullivan et al., "Isolation of putative progenitor endothelial cells for angiogenesis," Science, vol. 275, no. 5302, pp. 964-966, 1997.

[31] R. Samuel, L. Daheron, S. Liao et al., "Generation of functionally competent and durable engineered blood vessels from human induced pluripotent stem cells," Proceedings of the National Academy of Sciences of the United States of America, vol. 110, no. 31, pp. 12774-12779, 2013.

[32] R. A. Muzzarelli, M. El Mehtedi, C. Bottegoni, A. Aquili, and A. Gigante, "Genipin-crosslinked chitosan gels and scaffolds for tissue engineering and regeneration of cartilage and bone," Marine Drugs, vol. 13, no. 12, pp. 7314-7338, 2015.

[33] F. L. Mi, "Synthesis and characterization of a novel chitosangelatin bioconjugate with fluorescence emission," Biomacromolecules, vol. 6, no. 2, pp. 975-987, 2005. 
[34] Y. H. Lin, K. Y. Lu, C. L. Tseng, J. Y. Wu, C. H. Chen, and F. L. $\mathrm{Mi}$, "Development of genipin-crosslinked fucoidan/chitosan$\mathrm{N}$-arginine nanogels for preventing Helicobacter infection," Nanomedicine, vol. 12, no. 12, pp. 1491-1510, 2017.

[35] M. E. Frohbergh, A. Katsman, G. P. Botta et al., "Electrospun hydroxyapatite-containing chitosan nanofibers crosslinked with genipin for bone tissue engineering," Biomaterials, vol. 33, no. 36, pp. 9167-9178, 2012.

[36] K. Zafeiris, D. Brasinika, A. Karatza et al., "Additive manufacturing of hydroxyapatite-chitosan-genipin composite scaffolds for bone tissue engineering applications," Materials science \& engineering. C, Materials for biological applications, vol. 119, article 111639, 2021.

[37] A. Gilarska, J. Lewandowska-Łańcucka, K. Guzdek-Zając et al., "Bioactive yet antimicrobial structurally stable collagen/chitosan/lysine functionalized hyaluronic acid - based injectable hydrogels for potential bone tissue engineering applications," International Journal of Biological Macromolecules, vol. 155, pp. 938-950, 2020.

[38] S. Zeng, L. Liu, Y. Shi et al., "Characterization of silk fibroin/chitosan 3D porous scaffold and in vitro cytology," PloS one, vol. 10, no. 6, article e0128658, 2015.

[39] R. Langer and J. P. Vacanti, "Tissue engineering," Science, vol. 260, no. 5110, pp. 920-926, 1993.

[40] P. Sharma, P. Kumar, R. Sharma, V. D. Bhatt, and P. S. Dhot, "Tissue engineering; current status \& futuristic scope," Journal of Medicine and Life, vol. 12, no. 3, pp. 225-229, 2019.

[41] L. Geris and I. Papantoniou, "The third era of tissue engineering: reversing the innovation drivers," Tissue Engineering. Part A, vol. 25, no. 11-12, pp. 821-826, 2019.

[42] X. Wang, L. Gao, Y. Han et al., "Silicon-enhanced adipogenesis and angiogenesis for vascularized adipose tissue engineering," Advanced science, vol. 5, no. 11, article 1800776, 2018.

[43] X. Jing, H. Y. Mi, M. R. Salick, T. M. Cordie, X. F. Peng, and L. S. Turng, "Electrospinning thermoplastic polyurethane/graphene oxide scaffolds for small diameter vascular graft applications," Materials for biological applications, vol. 49, pp. 40-50, 2015.

[44] B. D. Holt, Z. M. Wright, A. M. Arnold, and S. A. Sydlik, "Graphene oxide as a scaffold for bone regeneration," Wiley interdisciplinary reviews: Nanomedicine and nanobiotechnology, vol. 9, no. 3, 2017.

[45] H. Zhou, Z. Wang, H. Cao et al., "Genipin-crosslinked polyvinyl alcohol/silk fibroin/nano-hydroxyapatite hydrogel for fabrication of artificial cornea scaffolds-a novel approach to corneal tissue engineering," Journal of Biomaterials Science. Polymer Edition, vol. 30, no. 17, pp. 1604-1619, 2019.

[46] F. Campos, A. B. Bonhome-Espinosa, G. Vizcaino et al., "Generation of genipin cross-linked fibrin-agarose hydrogel tissuelike models for tissue engineering applications," Biomedical materials, vol. 13, no. 2, article 025021, 2018.

[47] Y. Gong, Y. Yu, H. Kang et al., "Synthesis and characterization of graphene oxide/chitosan composite aerogels with high mechanical performance," Polymers, vol. 11, no. 5, 2019.

[48] S. Azizkhani, E. Mahmoudi, N. Abdullah, M. Ismail, A. W. Mohammad, and S. A. Hussain, "Synthesis and characterisation of graphene oxide-silica-chitosan for eliminating the $\mathrm{Pb}$ (II) from aqueous solution," Polymers, vol. 12, no. 9, p. 1922, 2020.

[49] Y. Zhang, M. Zhang, H. Jiang et al., "Bio-inspired layered chitosan/graphene oxide nanocomposite hydrogels with high strength and $\mathrm{pH}$-driven shape memory effect," Carbohydrate Polymers, vol. 177, pp. 116-125, 2017.

[50] T. Agarwal, R. Narayan, S. Maji et al., "Gelatin/carboxymethyl chitosan based scaffolds for dermal tissue engineering applications," International journal of biological macromolecules, vol. 93, no. Part B, pp. 1499-1506, 2016.

[51] Z. Gong and L. E. Niklason, "Blood vessels engineered from human cells," Trends in Cardiovascular Medicine, vol. 16, no. 5, pp. 153-156, 2006.

[52] E. Paz, Y. Ballesteros, J. Abenojar, J. C. Del Real, and N. J. Dunne, "Graphene oxide and graphene reinforced PMMA bone cements: evaluation of thermal properties and biocompatibility," Materials, vol. 12, no. 19n, 2019.

[53] M. Masyuk, A. Abduelmula, G. Morosan-Puopolo et al., "Retrograde migration of pectoral girdle muscle precursors depends on CXCR4/SDF-1 signaling," Histochemistry and Cell Biology, vol. 142, no. 5, pp. 473-488, 2014.

[54] K. Wang, X. Dai, J. He et al., "Endothelial overexpression of metallothionein prevents diabetes-induced impairment in ischemia angiogenesis through preservation of HIF- $1 \alpha / \mathrm{SDF}$ 1/VEGF signaling in endothelial progenitor cells," Diabetes, vol. 69, no. 8, pp. 1779-1792, 2020.

[55] G. Yu, P. Liu, Y. Shi, S. Li, Y. Liu, and W. Zhu, "Sitagliptin stimulates endothelial progenitor cells to induce endothelialization in aneurysm necks through the SDF-1/CXCR4/NRF2 signaling pathway," Frontiers in Endocrinology, vol. 10, p. 823, 2019.

[56] G. Odent Grigorescu, A. M. Rosca, M. B. Preda, R. Tutuianu, M. Simionescu, and A. Burlacu, "Synergic effects of VEGF-A and SDF-1 on the angiogenic properties of endothelial progenitor cells," Journal of Tissue Engineering and Regenerative Medicine, vol. 11, no. 11, pp. 3241-3252, 2017.

[57] D. Xue, E. Chen, H. Zhong et al., "Immunomodulatory properties of graphene oxide for osteogenesis and angiogenesis," International Journal of Nanomedicine, vol. Volume 13, pp. 5799-5810, 2018. 